$10 / 20-9698(1)$
5 SANDIA REPORT

SAND96-8237 - UC-406

Unlimited Release

Printed April 1996

BIBLIOGRAPHY OF PAPERS, REPORTS, AND PRESENTATIONS RELATED TO POINT-SAMPLE DIMENSIONAL MEASUREMENT METHODS FOR MACHINED PART EVALUATION

Jon M. Baldwin

Prepared by

Sandia National Laboratories

Abuquerque, New Mexico 87185 and Livermore, Calfornia 94551

for the Unied Stales Department of Energy

under Contract DE-AC04-94AL85000

Approved for public release; distribution is unlimiked. 
Issued by Sandia National Laboratories, operated for the United States Department of Energy by Sandia Corporation.

NOTICE: This report was prepared as an account of work sponsored by an agency of the United States Government. Neither the United States Government nor any agency thereof, nor any of their employees, nor any of the contractors, subcontractors, or their employees, makes any warranty, express or implied, or assumes any legal llability or responsibility for the accuracy, completeness, or usefulness of any information, apparatus, product, or process disclosed, or represents that its use would not infringe privately owned rights. Reference herein to any specific commercial product, process, or service by trade name, trademark, manufacturer, or otherwise, does not necessarily constitute or imply its endorsement, recommendation, or favoring by the United States Government, any agency thereof or any of their contractors or subconractors. The views and opinions expressed herein do not necessarily state or reflect those of the United States Government, any agency thereof or any of their contractors or subcontractors.

This report has been reproduced from the best available copy.

\section{Available to DOE and DOE contractors from:}

Office of Scientific and Technical Information

P. O. Box 62

Oak Ridge, TN 37831

Prices available from (615) 576-8401, FTS 626-8401

Available to the public from:

National Technical Information Service

U.S. Department of Commerce

5285 Port Royal Rd.

Springfield, VA 22161 
SAND96-8237

Unlimited Release

Printed April 1996

\title{
BIBLIOGRAPHY OF PAPERS, REPORTS AND PRESENTATIONS RELATED TO POINT-SAMPLE DIMENSIONAL MEASUREMENT METHODS FOR MACHINED PART EVALUATION
}

\author{
Jon M. Baldwin \\ Integrated Manufacturing Systems \\ Sandia National Laboratories/California
}

\begin{abstract}
This report is a compilation of results of a literature survey, conducted in support of an ongoing research program directed at developing methods for optimizing sampling and data analysis methods for point-sample techniques in dimensional metrology. Over 160 citations are included, with author abstracts where available.
\end{abstract}


a 


\section{BIBLIOGRAPHY OF PAPERS, REPORTS AND PRESENTATIONS RELATED TO POINT-SAMPLE DIMENSIONAL MEASUREMENT METHODS FOR MACHINED PART EVALUATION}

\section{Introduction}

The bibliographic information presented in this report has been compiled in the course of my involvement in and support of the Dimensional Inspection Techniques Specification (DITS) Project. It is presented here in the hope it will provide a useful entry point to the literature for others working in related areas.

DITS is an ongoing effort to produce tools and guidelines for optimum sampling and data analysis of machined parts, when measured using point-sample methods of dimensional metrology[1,2]. Primary among point-sample devices is the coordinate measuring machine, but neither DITS nor this literature survey is restricted to that particular implementation of the technology.

In compiling this literature survey, I have attempted to give bibliographic information for all references. In a few cases, primarily instances of internal or preliminary reports, complete information was not available. Where it seemed to me these reports might nevertheless be of interest, I have included the references in as complete form as possible. Whenever an author-generated abstract was available in computer-scannable format, it has been included. In a few cases, I have included my own comments intended to amplify or partially compensate for the lack of an abstract.

[1] C. W. Brown, Prec. Eng. 14(2, April), 110-111 (1992).

[2] K. D. Summerhays, R. P. Henke, J. M. Baldwin, R. M. Cassou, J. K. Finch, R. Detry, Dimensional inspection techniques specification - Planar face and datum features with addendum on opposite parallel plane features, R-95-QAP-01.,University of San Francisco, 2130 Fulton St., San Francisco, CA 94117-1080, 1994. 


\section{Publications}

Anbari,N; Trumpold,H (1989):

The influence of surface roughness in dependence of the probe ball radius measuring the actual size.

CIRP Sci. Tech. Comm. Rpt. (Surf. Met.).

Anon. (1994):

Mathematical Definition of Dimensioning and Tolerancing Principles.

ASME Y14.5.1M-1994, The American Society of Mechanical Engineers, NY.

Anon. (1994):

Dimensioning and Tolerancing.

ASME Y14.5M-1994, The American Society of Mechanical Engineers, NY.

Anon. (1989):

British standard guide to assessment of position, size, and departure from nominal form of geometric features.

BS7172:1989, British Standards Institution, Maynard Keynes, UK, 16 pp.

Comments:

Contains recommendations for feature parameterizations, sampling patterns and data processing for classical geometric features.

Anthony,GT; Anthony,HM; Bittner,B; Butler,B; Cox,M; Drieschner,R; Elligsen,R; Forbes,A; Gross,H; Hannaby,S; Harris,P; Kok,J (1993):

Chebyshev best-fit geometric elements.

NPL Report DITC 221/93, National Physical Laboratory, UK, September 1993. 


\section{Abstract}

This report summarizes the work carried out under the European Communities' Bureau of Reference (BCR) contract 3327/1/0/158/89/9-BCR-UK(30) Chebyshev Reference Software for the Assessment of Geometric Form. This involved developing algorithms and software to determine best fits in the Chebyshev sense to coordinate data nominally representing a line, plane, circle, sphere, cylinder or cone. Chebyshev is here extended to include maximum inscribed and minimum circumscribed circle, sphere and cylinder.

\section{Comments:}

Describes algorithms, implementation, testing.

\section{Anthony,GT; Cox,MG (1984):}

The design and validation of software for dimensional metrology.

NPL Report DITC 50/84, National Physical Laboratory, Teddington, Middlesex, UK, October 1984.

\section{Anthony,GT; Cox,MG (1985):}

Reliable algorithms for roundness assessment according to BS3730.

Proceedings of the Conference on Software for Co-ordinate Measuring Machines (pp 30-37) National Physical Laboratory, Teddington, Middlesex, UK.

\section{Boudreau,BD; Raja,J (1992):}

Analysis of lay characteristics of three-dimensional surface maps.

Int. J. Mach. Tools Manufact. 32(1/2), 171-177.

\section{Abstract:}

This paper describes a technique which can characterize the surface lay structure contained within a three-dimensional surface map. The technique is based on a generally known fact, that two, closely spaced, parallel profiles will appear shifted whenever the dominant lay direction is not perpendicular to the profile direction. The cross-correlation function is utilized to evaluate these apparent shifts. It will be shown that the functional relationship of these shifts is a characteristic of the lay pattern. Simple linear models are then applied to the shifts to quantify the lay characteristics. The resulting models can be used to extract lay angles and curvatures which correlate directly to the manufacturing process used to create the surface. 


\section{Bourdet,P; Lartigue,C; Leveaux,F (1993):}

Effects of data point distribution and mathematical model on finding the best-fit sphere to data.

Prec. Eng. 15(3, July), 150-157.

\section{Abstract:}

Probe calibration on a coordinate measuring machine is dependent on the accuracy with which the center of a reference sphere can be located. In this article, a theoretical model for sphere is fitted to a set of sampled surface points using the least-squares criterion. An analysis is conducted of various errors involved during the steps leading to the identification of the sphere and location of its center. The errors include those associated with surface accessibility for sampling, points sampling strategies, optimization algorithm, and probe diameter versus reference sphere diameter. Inferences are drawn regarding the relative influence of these errors, and a calibration strategy is proposed as a function of sampled points, optimization algorithm, and the geometric surface involved.

\section{Comments:}

Authors model center location errors with probing patterns and \#'s of points typical of common practice in probe calibration. Measurement errors are drawn from a uniform distribution on a fixed interval. Least squares fitting is used. Errors are plotted as a function of coverage. Experiments show reasonable agreement. They propose to improved the results by assuming a known reference sphere diameter. They do not say how they propose to supply the effective probe diameter.

\section{Brown,CW (1992):}

Dimensional inspection techniques for sample-point measurement technology.

Prec. Eng. 14(2, April), 110-111.

\section{Abstract:}

Today's industries face significant new challenges in the pursuit of precision manufacturing, higher quality products, and greater competitiveness. One specific challenge is attributed to the transition in the field of dimensional inspection and process control. This migration to computerized sample-point measurement equipment has resulted in the recognition of performance issues and underlying technological voids. The primary challenge is to overcome the issue of methods divergence. To rectify this problem, expanded knowledge must be obtained in technical areas of geometric tolerance representation, dimensional inspection 
techniques, and substitute geometry algorithms. The technology of sample-point measurements is based upon acquiring discrete coordinates on the surfaces of the workpiece of interest. Typically, sample-points are acquired by computer controlled measurement equipment, of which coordinate measuring machines (CMMs) represent the prime example. The computerized CMM requires software to drive the machine and to perform appropriate data analysis for feature extraction and tolerance evaluation. The transition being experienced in the emergence of, and increased reliance on, automated sample-point measurement equipment has focused attention on the method divergence performance issue. Methods divergence is the realization that dissimilar measurement methods yield different measured results for the same feature. Because of this, questions such as which measured value is correct, and which measured value represents the actual condition, are raised. As a result, today's practitioners of this technology cannot confidently be assured that measured results satisfy functional intent.

\title{
Brown,CW (1993):
}

Feature-based tolerancing for intelligent inspection process definition.

Proceedings of the 1993 International Forum on Dimensional Tolerancing and Metrology 27, 249-258.

\begin{abstract}
:
This paper describes a feature-based tolerancing capability that complements a geometric solid model with an explicit representation of conventional and geometric tolerances. This capability is focused on supporting an intelligent inspection process definition system. The feature-based tolerance model's benefits include advancing complete product definition initiatives (e.g., STEP - Standard for Exchange of Product model data), supplying computerintegrated manufacturing applications (e.g., generative process planning and automated part programming) with product definition information, and assisting in the solution of measurement performance issues. A feature-based tolerance information model was developed based upon the notion of a feature's toleranceable aspects and describes an objectoriented scheme for representing and relating tolerance features, tolerances, and datum reference frames. For easy incorporation, the tolerance feature entities are interconnected with STEP solid model entities. This will explicitly represent the tolerance specification for mechanical products, support advanced dimensional measurement applications, and assist in tolerance-related methods divergence issues.
\end{abstract}




\section{Burdekin,M; Pahk,HJ (1989):}

The application of a microcomputer to the on-line calibration of the flatness of engineering surfaces.

Proc. Instn. Mech. Engrs. 203, 127-137.

\section{Abstract:}

In this paper, a microcomputer aided system for the calibration of the flatness of engineering surfaces is proposed. Rectangular grid-type measurement procedures with closing error technique are offered for angular assessment on the surfaces using electronic precision level. A new technique called "enclose tilt technique" (ETT) is developed for the analysis of flatness in terms of minimum zone, and defines the flatness in terms of British and ISO standards. These measurement analysis processes are performed on-line with a microcomputer using an analogue/digital interface, amplifier with low-pass filter, triggering technique, etc., and appropriate high-level and low-level programming language. A practicál flatness calibration of a granite table has been performed for application, and the results are shown with up to 0.1 um uncertainty. The effect of the weight of the precision level, and the rigidity test of the granite table have also been assessed.

\section{Comments:}

Sampling patterns discussed are more appropriate to determination of long-period errors of surface plates than to machined part metrology.

\section{Butler,BP; Forbes,AB; Harris,PM (1994):}

Algorithms for geometric tolerance assessment.

NPL Report DITC 228/94, National Physical Laboratory, Teddington, Middlesex, UK.

Design and tolerance information can be stated in terms of nominal form, parameter constraints, and form constraints involving measured data points. The tolerance assessment problem is to find a set of parameter values that satisfy the constraints, and this may be achieved by formulating and solving an associated optimisation problem. In this report we describe how to exploit standard optimisation software in order to solve this optimisation problem. Our intention is to describe generic harnesses or templates that act as interfaces between the tolerance assessment problem and the optimisation software. The harnesses are specified using Matlab although, since their design is transparent, translation into other languages should be straightforward. We illustrate the application of these submodules to a number of typical tolerance assessment problems. 


\section{Comments:}

Really nice demonstration of how to use canned software to solve a variety of metrology problems.

\section{Cardou,A; Bouillon,G (1977):}

Surface plate flatness definition through linear programming.

Microtechnic 31, 48-50.

\section{Cardou,A; Bouillon,G; Tremblay,G (1972):}

Some considerations on the flatness of surface plates.

Microtechnic 26(7), 367-368.

\section{Carpinetti,LCR; Chetwynd,DG (1994):}

A new strategy for inspecting roundness features.

Prec. Eng. 16(4, October), 283-289.

Abstract:

This article addresses the problem of inspection of roundness error (in contrast to the measurement of roundness error) and the idea of GO-NOGO inspection software. A new approach is presented in which the formalism of linear programming is applied through reexpressing the initial geometrical problem and adding further constraints. The ultimate position is to provide a set of constraints from which the required inspection information can be obtained by examining only the feasibility of the mathematical program, with no need to consider its iteration toward optimality. Feasibility may be explored using an existing, although not widely known, algorithm to guide the GO-NOGO decision. The basis of the algorithm is given along with practical examples of how it works. Tests indicate that on real data it usually involves a computational effort only modestly higher than using an exchange algorithm to find an optimal fit. Thus, it appears quite practical for applications to the relatively small computer of a standard instrument. 


\title{
Carr,KM (1995):
}

Modeling and verification methods for the inspection of geometric tolerances using point data.

Ph.D. Dissertation, University of Illinois, Urbana-Champaign. 189+xvi p.

\begin{abstract}
:
The ability to specify, produce, and verify parts with sufficiently small variations such that they are functionally equivalent is vital to the success of mass production. The amount of allowable variation in a part is specified by tolerances. To ensure a part conforms to these tolerances, inspection methods are required. With the increase demand for flexibility on the factory floor, a programmable inspection tool, known as the coordinate measuring machine $(\mathrm{CMM})$, has become popular. A CMM is programmed to collect point data information from the surface of a part. This data is analyzed by verification algorithms to determine if the part conforms to the tolerance specifications. Since CMMs determine the conformance of a part based on point data information, methods for reasoning about tolerances based on this type of information are required. In this work, a framework is developed for reasoning about the inspection of geometric tolerances, which include form, size, orientation, and position tolerances. Methodologies are developed that give geometric and mathematical characterizations of tolerance zones. The geometric characterization provides an understanding of the shape of the zone and how it is allowed to move in space. The mathematical characterization defines a set of equations that the parameters of the zone and all enclosed points must satisfy. These characterizations provide a basis for defining search/optimization models of the inspection of geometric tolerances that can be solved using standard optimization techniques. The ability of this framework to model the inspection of the commonly used geometric tolerances is demonstrated. The resulting verification algorithms are based on min-max optimization models and represent an important departure from current verification methods, which are based on least-squares algorithms. The algorithms developed in this work are shown to be correct, robust, stable, efficient, and capable of improving upon the least-squares solution by as much as twenty percent. This improvement can result in significant cost savings in mass production. With the improvement of part quality as the main motivation behind all efforts to understand, measure, and eliminate deviations in the manufacturing of parts, this framework represents an important step forward in the inspection of geometric deviations.
\end{abstract}




\section{Carr,K; Ferreira,P (1995):}

Verification of form tolerances. Part I: Basic issues, flatness and straightness.

Prec. Eng. 17(2), 131-143.

\section{Abstract:}

The ANSI Y14.5 National Standard on Dimensioning and Tolerancing definition for form tolerances requires the form error of a surface to be less than some set limit. However, most inspectors are interested in the minimum form error, known as the minimum zone solution. To compute the minimum zone flatness, an algorithm must determine the minimum distance between two parallel planes so that all data points are between the two planes. Therefore, the minimum zone solution minimizes the maximum error between the data points and a reference plane. Current coordinate measuring machine verification algorithms are based on the least-squares solution, which minimizes the sum of the squared errors, resulting in a possible overestimation of the form tolerance. Therefore, while coordinate measuring machine algorithms successfully reject bad parts, they may also reject some good parts. The verification algorithms developed in this set of papers compute the minimum zone solution of a set of data points sampled from a part. Computing the minimum zone solution is inherently a non-linear optimization problem. The proposed algorithms solve a sequence of linear programs that converge to the solution of the nonlinear problem. The linear programs result from a novel combination of coordinate and scaling transformations and do not change the original optimization problem. Therefore, given adequate initial conditions, the sequence of linear programs will converge to the minimum zone solution. Implementation and testing results demonstrate the correctness of these formulations. The implementation of these verification algorithms in a production environment can reduce the possibility of rejecting good parts, thereby reducing costs.

\section{Comments:}

Solves minimum zone for a given data set. Algorithm solves a series of linear optimization programs that converge to the solution of the nonlinear problem. Linear programs result from combination of coordinate and scaling transformations and do not change the original optimization problem. This paper deals with flatness \& straightness. Does not address sample point selection. Offers a definition of a verification method, that allows use of point sample data, but does not specify the manner in which it is employed. Methods presented do, in fact, apply minimum zone to the unmodified data set. 


\section{Carr,K; Ferreira,P (1995):}

Verification of form tolerances. Part II: Cylindricity, circularity and straightness of a median line.

Prec. Eng. 17(2), 144-156.

\section{Abstract:}

Most inspectors measure form tolerances as the minimum zone solution, which minimizes the maximum error between the data points and a reference feature. Current coordinate measuring machines verification algorithms are based on the least-squares solution, which minimizes the sum of the squared errors, resulting in a possible overestimation of the form tolerance. Therefore, although coordinate measuring machines algorithms successfully reject bad parts, they may also reject some good parts. The verification algorithms developed in this set of papers compute the minimum zone solution of a set of datapoints sampled from a part. Computing the minimum zone solution is inherently a nonlinear optimization problem. This paper develops a single verification methodology that can be applied to the cylindricity and straightness of a median line problems. The final implementable formulation solves a sequence of linear programs that converge to a local optimal solution. Given adequate initial conditions, this solution will be the minimum zone solution. This methodology is also applied to the problems of computing the minimum circumscribed cylinder and the maximum inscribed cylinder. Experimental evidence that the formulations are both robust and efficient is provided.

\section{Comments:}

See remarks on part I article. Gives methods for minimum zone solutions for cylindricity, circularity and straightness of a median line, also for computation of minimum circumscribed and maximum inscribed cylinders.

\section{Caskey,G; Hari,Y; Hocken,R; Machireddy,R; Raja,J; Wilson,R; Zhang,G; Chen,K; Yang,J (1992):}

Sampling techniques for coordinate measuring machines.

Proceedings of the 1991 National Science Foundation Design and Manufacturing Systems Conference; January; 779-786.

Abstract:

The purpose of this project was to systematically examine the interaction between the various procedures involved in measurement of mechanical parts using coordinate measuring 
machines (CMMs). We have examined the systematic and random errors in coordinate measuring, the sampling methodologies appropriate to different part geometries, and the algorithms used to analyze the coordinate data to produce mathematical descriptions of geometric entities.

\title{
Comments:
}

Describes the evolution of their least squares and minimax fitting procedures, testing against PTB data sets and other synthetic data, sampling strategy studies for planar features. Results are reported for "stratified" sampling patterns. Also discusses state of their work on CMM and process characterization.

\section{Chetwynd,DG (1979):}

Roundness measurement using limacons.

Prec. Eng. 1, 137-141.

\section{Chetwynd,DG (1985):}

Applications of linear programming to engineering metrology.

Proc. Instn. Mech. Engrs. 199(B2), 93-100.

\begin{abstract}
:
Computer-based metrology now makes use of exchange algorithms for computing best-fit geometries in which the solution is obtained by a series of iterations each involving the exchange of one previously unused data point for one of the dominant points of the previous iteration according to formal rules. While conferring large advantages in specific circumstances, their major significance is that of being examples of a class of optimization of much wider applicability. This paper examines the theoretical basis of these algorithms in linear programming and develops a general approach to the solution of this class from which new applications can be derived. To maintain an engineering context in the analysis, practical examples are used, mainly from the field of roundness measurement.
\end{abstract}

\section{Chetwynd,DG (1985):}

Linearized exchange algorithms in metrology.

National Physical Laboratory, UK; Proceedings of the Conference on Software for Coordinate Measuring Machines; pp 24-29. 


\title{
Chizhik,SA; Gorbunov,VV; Myshkin,NK (1995):
}

Analysis of molecular scale roughness effect on contact of solids based on computer modeling.

Prec. Eng. 17(3, July), 186-191.

\section{Abstract:}

The real contact between solids is modeled at the molecular scale, and the results are presented. Adhesion, real contact area, and approach of the surfaces are evaluated with the help of scanning, tunneling, and atomic force microscope imaging of the surfaces.

\section{Cox,MG (1981):}

The least squares solution of overdetermined equations having band or augmented band structure.

IMA J. Numer. Anal. 1, 3-22.

\section{Cox,MG (1985):}

Software for computer-aided measurement.

Lab. Practice 34, 59-63.

\section{Cox,MG (1992):}

Improving CMM software quality.

NPL DITC 194/92, National Physical Laboratory, Teddington, Middlesex, UK, 8 pp.

\begin{abstract}
:
The enumerate methodologies for coordinate measurement machine (CMM) software conformance testing and then concentrate on one in particular, viz. the use of reference data sets, or soft gauges. The advantages of such an evaluation mechanism are identified and discussed. Progress on a draft standard concerned with the use of reference data sets for conformance testing of CMM software is described, as is preliminary work on a method of operation of a third party CMM software conformance testing service. Related activity is reported. This includes work on reference software for CMMs, European CMM software intercomparisons and further relevant standardization. Finally, the importance of software traceability is emphasised.
\end{abstract}




\section{Cox,MG; Forbes,AB (1992):}

Strategies for testing form assessment software.

NPL Report DITC 211/92, National Physical Laboratory, Teddington, Middlesex, UK.

\section{Abstract:}

This report describes methods for generating test data sets and reference results which can be used to test assessment software employed in coordinate metrology for finding leastsquares best-fit curves and surfaces to data. The methods do not rely on the use of reference software but instead exploit known mathematical properties which hold at the solution of the data approximation problem. The methods are flexible, straightforward to implement and should be of value in designing conformance testing procedures. The methods are illustrated for the problem of finding the least-squares best-fit circle to data.

\section{Cox,MG; Jones,HM (1988):}

An algorithm for least-squares circle fitting to data with specified uncertainty ellipses.

NPL Report DITC 118/88, National Physics Laboratory, Teddington, Middlesex, UK. (also to appear in IMA J. Numer. Anal.)

\section{Dawson,DJW (1992):}

Cylindricity and its measurement.

Int. J. Mach. Tools Manufact. 32(1/2), 247-253.

\section{Abstract:}

Out-of-cylindricity has always been a useful geometric parameter and its use is becoming more widespread. Along with this increase in use, has been an increasing awareness of the limitations of current instrumentation in measuring cylindricity. This paper will analyze the limitations of current instrumentation and measurement techniques with respect of cylindricity. The paper also suggests areas for both addressing these limitations, whilst also developing the use of the parameter.

\section{Comments:}

Regards cylindricity as zone of minimum radial separation and regards accuracy of available apparatus to be the main current problem. Catalogs areas of applicability for maximum inscribed, minimum circumscribed, minimum zone and least squares fits. As significant 
limitations lists possible nonuniqueness of MI \& MC solutions, instability of MI \& MC solutions in presence of taper, data sample sensitivity of all but least squares solution, failure to reveal spatial character of form deviation.

\section{de Bruin,W; Meijer,J (1980):}

Analysis of flatness measurement and form stability of a granite surface plate.

Ann. CIRP 29(1), 385-390.

\section{Abstract:}

It is rather difficult to compare quantitatively flatness measuring results of one and the same object (e.g. in case of a long-term stability investigation) or the results of a series of products (e.g. from the same machine tool in order to indicate machine tool influences). An extension of a computer program applied to the evaluation of flatness measurement (as described in the CIRP Annals, vol. 26 (1977) no.1), separates the form deviations of a surface into a geometrical part and a random part. The geometric part consists of a best-fitting, non-flat, reference plane, composed of the single curvature of a sphere and the double curvature of a real twisted plane. Three parameters (the dimensionless curvature parameters of sphericity and torsion, together with the direction of the torsion vector) are able to characterize the geometrical part of the surface; a fourth one is a measure for the random part. Advantages of the method are demonstrated with the aid of a long-term investigation into the form stability of a granite surface plate. Another extension of the program makes it now possible to measure surfaces with non-rectangular contours in the same way as it is being done in case of a rectangular surface plate.

\section{deBruin,W; Meijer,J; Nawijn,A (1977):}

Surface plate measurements.

Ann. CIRP 26(1), 251-255.

\section{Del Taglia,A; Vignalo,M (?):}

Analysis of cylindrical surfaces for automatic diagnosis of manufacturing errors.

Department of Mechanical and Industrial Technology, Universita di Firenze, Firenze, Italy.

\section{Abstract:}

The aim of this work was to analyze cylindricity errors so that the effect of the manufacturing process on workpiece defects would be easily decipherable. Using a predefined reference 
cylinder, the total cylindricity error was broken down into three simple error categories, i.e., axis straightness error, generatrix straightness error, and normal cross section error. These were computed using several cross sections of the workpiece surface acquired by a roundness error meter interfaced to a personal computer. Special software was developed to automate the test procedures, data acquisition and processing, and error computation. With this new system, all major functions are completely automatic and, in addition, measurements may be programmed in cycles and carried out unattended.

\section{Comments:}

Explores relation of form error to size and position errors. Attempt to link to error sources in manufacturing process.

\section{DeVries,WR; Li,C-J (1985):}

Algorithms to deconvolve stylus geometry from surface profile measurements.

ASME Trans., Journal of Engineering for Industry 107(May), 167-174.

\section{Abstract:}

Stylus methods for measuring surface topography impose certain limits on resolving the true profile of a surface. These limits are primarily due to the finite geometric size of the stylus. In this paper, kinematic and geometric algorithms are developed to compensate for stylus geometry so as to present a better picture of the true surface profile. To demonstrate their effectiveness, these algorithms are applied to simulated deterministic and stochastic profile measurements.

\section{Comments:}

Sinusoidal and Gaussian surfaces are considered. Does not consider dynamic effects nor surface deformation under probing force. Correction algorithms require first (and one the second) derivative of the surface.

\section{Dhanish,PB; Shunmugam,MS (1991):}

An algorithm for form error evaluation - using the theory of discrete and linear Chebyshev approximation.

Comp. Meth. Appl. Mech. Eng. 92, 309-324. 


\section{Abstract:}

ISO defines form error based on the concept of minimum zone, but does not describe the methods to obtain the same. In this paper, drawbacks of the available methods are discussed and a new algorithm is presented. This algorithm is guaranteed to give the minimum value of form error. The algorithm is well-suited for implementation on a computer which may be interfaced with a Coordinate Measuring Machine.

Comments:

Presents a general algorithm for evaluation of minimum zone form error. Claimed to guarantee true minimum value. Considers only measured points.

\section{Ehmann,KF; Hong,MS (1994):}

A generalized model of the surface generation process in metal cutting.

CIRP Annals 43(1, Manufacturing Technology), 483-486.

\section{Abstract:}

Machined surfaces are generated by a variety of processes, each of which produces a surface with its own characteristic topography. A method for the prediction of the topography of the generated surfaces has been developed based on general models of the machine tool's kinematics and a generalized model of deterministic and non-deterministic cutting tool geometries. The model termed the surface-shaping system accounts for not only the nominal or global motions of the machine but also takes into account errors during machining such as tool runout, machine deformation and vibration, as well as higher order motions. Based on the surface shaping system model a computer simulation system has been developed which facilitates the 3D graphical representation and evaluation of the topography of the generated surface.

\section{ElMaraghy,WH; EIMaraghy,HA; Wu,Z (1990):}

Determination of actual geometric deviations using coordinate measuring machine data.

Manufacturing Review 3(March), 32-39.

\section{Abstract:}

Focuses on the development of a procedure and algorithms for the systematic comparison of geometric deviations of measured features with their specified geometric tolerances. To automate the inspection of mechanical parts, it is necessary to analyze the measurement data captured by coordinate measuring machines in order to detect out-of-tolerance 
conditions. A procedure is presented for determining the geometric tolerances from the measured 3-dimensional coordinates on the surface of a cylindrical feature. This procedure follows the definitions of the geometric tolerances used in the current standards, and is capable of determining the value of each geometric deviation from the composite 3D data. The developed algorithms adopt the minimum deviation zone criterion. Nonlinear numerical optimization techniques are used to fit the data to the minimum tolerance zone. Two test cases are given which demonstrate the successful determination of geometric deviations from given simulated data.

\section{EIMaraghy,WH; Wu,Z; EIMaraghy,HA (1989):}

Evaluation of actual geometric tolerances using coordinate measuring machine data.

Advances in Design Automation - 1989, ASME Design Technical Conferences - 15th Design Automation Conference, Montreal, Que, Can, Sep 17-21.

\section{Abstract:}

This paper focuses on the development of a procedure and algorithms for the systematic comparison of geometric variations of measured features with their specified geometric tolerances. To automate the inspection of mechanical parts, it is necessary to analyze the measurement data captured by coordinate measuring machines (CMM) in order to detect out-of-tolerance conditions. A procedure for determining the geometric tolerances from the measured three dimensional coordinates on the surface of a cylindrical feature is presented. This procedure follows the definitions of the geometric tolerances used in the current Standards, and is capable of determining the value of each geometric tolerance from the composite 3-D data. The developed algorithms adopt the minimum tolerance zone criterion. Nonlinear numerical optimization techniques are used to fit the data to the minimum tolerance zone. Two test cases are given in the paper which demonstrate the successful determination of geometric tolerances from given simulated data.

\section{Endres,WJ; DeVor,RE; Kapoor,SG (1995):}

A dual-mechanism approach to the prediction of machining forces, Part 1: Model development.

ASME Transactions Journal of Engineering for Industry 117, 526-533.

\section{Abstract:}

A cutting-process model addressing the chip removal and edge ploughing mechanisms separately yet simultaneously is presented. The model is developed such that it is readily applicable in an industrial setting, its coefficients have physical meaning, and it can be 
calibrated with a concise quantity of orthogonal cutting data. The total cuttting and thrust forces are each the summation of its individual components acting on the rake face and clearance face. These components are calculated using the rake and effective clearance angles from the normal and friction forces acting on each of these tool surfaces. These normal and friction forces are calculated by the chip removal and edge ploughing portions of the model, respectively, using four empirical coefficients. To calculate the clearance face forces, the interference volume is required, the calculation of which is based on a geometrical representation of the clearance face interference region. This representation is characterized in part by the depth of tool penetration, which is influenced by thermal energy generation and is therefore determined using a fifth empirical model.

\section{Endres,WJ; DeVor,RE; Kapoor,SG (1995):}

A dual-mechanism approach to the prediction of machining forces, Part 2: Calibration and validation.

ASME Transactions Journal of Engineering for Industry 117, 534-541.

\section{Abstract:}

The Dual-Mechanism Machining Force Model (DMMFM) developed in part 1 of this paper is calibrated through a specially developed algorithm, then validated. The calibration results are used to study the total machining force predictive capabilities of both the traditional lumped shearing model and the DMMFM. It is shown that the Dual-Mechanism Approach contributes greatly to our ability to both physically explain the trends in the machining force data and to understand their implications. This is achieved through an interpretation of the individual rake face and clearance face forces that are predicted using the DMMFM. The interpretation is based on the relations of these rake face and clearance face forces to the process inputs resulting from their effects on the DMMFM coefficients through thermal energy generation and temperature, shear- strain level and shear-strain rate. Some implications of the knowledge of the individual rake face and clearance face forces, as predicted by the DMMFM, are also discussed.

\section{Estler,WT; Phillips,SD; Borchardt,B; Hopp,T; Witzgall,C; Levenson,M; Eberhardt,K; McClain,M; Shen,Y; Zhang,X (1995):}

Error compensation for CMM touch trigger probes. Proceedings of the 10th Annual Meeting of the American Society for Precision Engineering, Austin, Texas, 428-431. 


\title{
Comments:
}

Presents a kinematically-based model of pretravel variation in switching CMM probes. Model is successful in expressing pretravel variation as a function of polar and azimuthal angles of incidence.

\section{Etesami,F; Qiao,H (1990):}

Analysis of two-dimensional measurement data for automated inspection.

Journal of Manufacturing Systems 9(1), 21-34.

\begin{abstract}
:
Automatic sensing machines often provide a mass of geometric boundary information that has to be analyzed in order to estimate desired geometric parameters of part features. This problem has been approached primarily by curve fitting and often ad-hoc and arbitrary definitions of geometric dimensioning and tolerancing terms such as "size" and "position". As indicated in ANSI and ISO standards and as evident to designers, parts do not make contact on best fit surfaces but on their extreme functional boundaries. In this paper, definitions based on computational geometry are given to formalize the meaning of geometric imperfections according to the implications of the standards. Given two-dimensional data point patterns, the problem of determining the size, form, orientation, and position of circular, planar, and parallel plane features are formally expressed. Those definitions which lead to known solution techniques in computational geometry are identified. Solutions to other problems not found in related studies are presented, such as the roundness problem and the size of parallel-plane features. A formal technique for dealing with datum related features is also presented. Finally, an example is given that illustrates how a typical position tolerance can be verified with a simple procedure. The application of these techniques is demonstrated in automated dimensional inspection and in modeling of manufactured part geometries.
\end{abstract}

\section{Feng,SC; Hopp,TH (1991):}

A review of current geometric tolerancing theories and inspection data analysis algorithms.

NISTIR-4509, National Institute of Standards and Technology, Gaithersburg, MD.

\section{Abstract:}

This report provides an overview of the state of the art in mechanical dimensioning and tolerancing theories and CMM inspection data analysis technology. We expect that the information included in this review will benefit CMM software developers, CMM users, and researchers of new CMM technology. This document is the result of a survey of published 
geometric dimensioning and tolerancing theories and post-inspection data analysis algorithms. Both traditional and modern theories have been reviewed. Principles on which current national standards and international standards (are) based have been stated. These geometric dimensioning and tolerancing principles are commonly used in mechanical design and part inspection. Post-inspection data analysis algorithms, used for extracting features and evaluating tolerances, have also been reviewed. The effects of using different fitting criteria are discussed. From this theory and algorithm review, we recommend directions for future development in these areas. The bibliography covers activities and accomplishments of the research in advancing inspection technology.

Forbes, $\mathrm{AB}$ (1989):

Least-squares best-fit geometric elements.

NPL-DITC-140/89, National Physical Laboratory, Teddington, England.

\title{
Abstract:
}

The aim of the report is to describe algorithms for finding least-squares best-fit geometric elements to data. Such data fitting problems arise in coordinate metrology where it is required to assess a manufactured workpiece using data gathered by a coordinate measurement system. The elements considered are lines, planes, circles, spheres, cylinders and cones. The algorithms employ stable parameterizations of the elements and are based on sound mathematical and numerical principles.

Forbes,AB (1992):

Geometric tolerance assessment.

NPL Report DITC 210/92, National Physical Laboratory, Teddington, Middlesex, UK.

\begin{abstract}
:
Tolerance assessment is a key step in maintaining and improving quality in manufactured products. This report describes how a class of design and tolerance specifications can be formulated in terms of parameterized curves and surfaces and parameter and form constraints. The tolerance assessment problem can then be addressed by optimization techniques. It is shown how many important tolerance assessment problems can be formulated as simple matching problems for which efficient solution algorithms can be developed.
\end{abstract}


Forbes, $\mathrm{AB}$ (1994):

Validation of software for dimensional metrology.

NPL Report DITC 225/94, National Physical Laboratory, Teddington, Middlesex, UK.

Abstract:

In this paper we discuss three questions relating to the use of reference data sets and reference results in black box tests for validating assessment software: I) how to generate data and results, ii) how to represent solutions in a stable way and iii) how to compare test results and reference results. We describe a general method for generating data and results which goes some way to addressing all three problems and illustrate the concepts introduced for least squares form assessment and theodolite triangulation.

Frank, RN (1992):

Coordinate measuring machine technology and its application to the analysis of ANSI Y-14.5 criteria.

UCRL-JC-109607, Lawrence Livermore National Laboratory, 68 pp, Interagency Mechanical Operations Group (IMOG) gauging subgroup conference, Kansas City, MO (United States), 7 Apr.

Abstract:

A Coordinate Measuring Machine (CMM) is an electromechanical device which is used to analyze the geometric condition of precision machined mechanical parts. Measurements are made by contacting the surface of parts with a sensing device (probe). This creates an X-Y-Z data point. Evaluations of geometric condition and compliance with ANSI Y14.5 drawing specifications are made through mathematical analysis of sets of data points. CMM systems are becoming the primary for the inspection of precision machined mechanical components. Most CMM systems, however, do not analyze the data which they gather in accordance with Y14.5 definitions. This text presents an overview of CMM technology and some examples of fundamental methods, through which ANSI Y14.5 requirements may be satisfied.

\section{Fujii,Y; Iwabe,H (1985):}

Effect of dynamic behavior of end milling on working accuracy - Mechanism of generating shape error of bottom surface.

Bull. Japan. Soc. Prec. Eng. 19(4, December), 297-299. 
Fukuda,M; Shimokohbe,A (1984):

Algorithms for form error evaluation - methods of the minimum zone and the least squares.

Proc. Intern. Symp. Metrol. Qual. Cont. Prod., 197-202.

\section{Abstract:}

Form errors evaluations are specified by ISO in the minimum zone concept. However, no sophisticated method is available to numerically determine the form errors on the basis of the minimum zone evaluation. This report introduces an algorithm for the minimum zone evaluation by minimax approximation and makes a comparison between this method and the least squares method in terms of evaluation time and accuracy.

\section{Comments:}

Their approach is to approximate the minimum zone solution by taking the constructing circumscribing features parallel to the minimax best fit feature.

\section{Goto,M; Iizuka,K (1977):}

An analysis of the relationship between minimum zone deviation and least squares deviation in circularity and cylindricity.

Proc. Intern. Conf. Prod. Eng., ?61-?70.

\section{Greenwood,JA; Williamson,JBP (1966):}

Contact of nominally flat surfaces

Proc. Roy. Soc. London 295A, 300-319.

\section{Abstract:}

It is usually assumed that the real area of contact between two nominally flat metal surfaces is determined by the plastic deformation of their highest asperities. This leads at once to the result that the real area of contact is directly proportional to the load and independent of the apparent area--a result with many applications in the theories of electrical contacts and friction. Archard pointed out that plastic deformation could not be the universal rule, and introduced a model which showed that, contrary to earlier ideas, the area of contact could be proportional to the load even with purely elastic contact. This paper describes a new theory of elastic contact, which is more closely related to real surfaces than earlier theories. We show how the contact deformation depends on the topography of the surface, and establish 
the criterion for distinguishing surfaces which touch elastically from those which touch plastically. The theory also indicates the existence of an 'elastic contact hardness', a composite quality depending on the elastic properties and the topography, which plays the same role in elastic contact as the conventional hardness does in plastic contact. A new instrument for measuring surface topography has been built; with it the various parameters shown by the theory to govern surface contact can be measured experimentally. The typical radii of surface asperities have been measured. They were found, surprisingly, to be orders of magnitude larger than the heights of the asperities. More generally we have been able to study the distributions of asperity heights and of other surface features for a variety of artifacts prepared by standard techniques. Using these data we find that contact between surfaces is frequently plastic, as usually assumed, but that surfaces which touch elastically are by no means uncommon in engineering practice.

\section{Harlan,R; Nasson,Mark A (?):}

Tolerancing small segments of spheres and cylinders.

CSDL-C-5785, Charles Stark Draper Laboratory, Cambridge, MA, 13 pp.

\section{Hirst,W; Hollander,AE (1974):}

Surface finish and damage in sliding

Proc. Roy. Soc. London, A 337, 379-394.

Abstract:

The ability of rubbing surfaces to support load depends on the surface finish. Many types of surface used in engineering have a random structure; their profile may be represented by the waveform of a random signal defined by two parameters sigma and beta-star describing the height distribution and the autocorrelation function respectively. Surfaces covering a range of values of both sigma and beta-star have been prepared and for each surface the ability to withstand damage in conditions of boundary lubrication has been determined. It is shown that the values of sigma and beta-star taken together provide good criteria for assessing this property. For the rougher surfaces a sufficient criterion is the ratio sigma/beta-star. Very smooth surfaces, however, fail readily, and when beta-star is very small all surfaces fail regardless of the value of sigma. 


\title{
Hocken,RJ; Raja,J; Babu,U (1993):
}

Sampling issues in coordinate metrology.

Proceedings of the 1993 International Forum on Dimensional Tolerancing and Metrology 27, 97-111.

\begin{abstract}
:
Coordinate measuring machines acquire data one point at a time on the surfaces of the parts being measured. These data must be analyzed to produce a substitute geometry to be compared to the design intent. Algorithms are used to analyze these data. The results obtained are strongly dependent upon measuring errors, the form error of the part, the sampling strategy, and the density of points sampled. In this paper we review these problems and discuss some of the progress addressing them during the past few years.
\end{abstract}

\section{Comments:}

Review of the effect of measuring errors, form error, sampling strategy and point density on CMM measurement results. Primarily considers "best" patterns of uniform sampling. Consider mainly computer-generated data sets. Systematic form errors chosen are not always particularly real. Good literature review. Circle data is basically that used in DITS report. Their approach in general is along those same lines.

\section{Hong,MS; Ehmann,KF (1995):}

Three-dimensional surface characterization by two-dimensional autoregressive models.

ASME Trans. J. Tribology 117(July), 385-391.

\section{Abstract:}

An attempt is made to characterize and synthesize engineering surfaces. The proposed method, based on two-dimensional difference equations and two-dimensional autoregressive models, is not only an analytical tool to characterize but also to generate/synthesize threedimensional surfaces with desired properties. The developed method expresses important three-dimensional surface characteristics such as the autocorrelation or power spectrum density functions in terms of the two-dimensional autoregressive coefficients. 
Hopp,TH (1993):

Computational metrology.

Proceedings of the 1993 International Forum on Dimensional Tolerancing and Metrology 27, 207-217.

\section{Abstract:}

Coordinate measuring systems (CMSs) assess length-based characteristics of mechanical parts by measuring points on the part surface and analyzing the point data. Data analysis software can contribute significantly to the total measurement error of a CMS. Factors affecting software performance include the choice of analysis method, the quality of the software, and characteristics of the specific measurement task. By computational metrology we mean the study of how data analysis factors affect the measurement uncertainty of a CMS. This paper describes research at NIST on computational metrology. Metrological questions fall into two categories: (1) the proper choice of data analysis objective for a particular application and (2) the performance of the implementation. Our research goal is to develop the basis for a national standard in the U.S. for CMS software performance evaluation. We are implementing a Special Test service to be offered through the NIST Measurement Services Program. The service is based on a black-box model of software, in which the internal structure of the software and the choice of solution methods are assumed to be unknown. The model identifies a number of error sources for data analysis software. We are designing test methods for identifying the various components of the model and how those components relate to measurement uncertainty in inspection applications.

\section{Hopp,TH; Levenson,MS (1995):}

Performance measures for geometric fitting in the NIST algorithm testing and evaluation program for coordinate measurement systems

NIST Journal of Research 100(September-October), 563-574.

\section{Abstract:}

The Algorithm Testing and Evaluation Program for Coordinate Measurement Systems (ATEP-CMS) is a Special Test Service offered under the NIST Calibration Program. ATEP-CMS evaluates the performance of geometric fitting software usedevaluates the performance of geometric fitting software used in coordinate measurement systems. It is a Special Test because it is a new type of NIST service, experimantal in nature and unsupported by historical data. This report documents and explains the rationale of the performance measures used in ATEP-CMS and analyzes the uncertainties of those measures. 


\section{Huang,ST; Fan,KC; Wu,JH (1993):}

A new minimum zone method for evaluating straightness errors.

Prec. Eng. 15(3, July), 158-165.

\section{Abstract:}

A new minimum zone method for straightness error analysis is proposed in this article. Based on the criteria for the minimum zone solution and strict rules for data exchange, a simple and rapid algorithm, called the control line rotation scheme, is developed for the straightness analysis of planar lines. Extended works on the error analysis of spatial lines by the least parallelepiped enclosure are also described. Some examples are given in terms of the minimum zone and least-squares. Finally, this easy-to-use method is illustrated by an example that demonstrates that, for a planar line, the minimum zone solution can even be found without the use of a computer.

\section{Huang,ST; Fan,KC; Wu,JH (1993):}

A new minimum zone method for evaluating flatness errors.

Prec. Eng. 15(1, January), 25-32.

\section{Abstract:}

A new minimum zone method for flatness error analysis is proposed in this article. Based on the criteria for the minimum zone solution and strict rules for data exchange, a simple and rapid algorithm, called the control plane rotation scheme, is developed for the flatness analysis of a flat surface. Experimental work was performed, and some examples are given in terms of the minimum zone and least squares solutions.

\section{Hurt,JJ (1980):}

A comparison of several plane fit algorithms.

Ann. CIRP 29(1), 381-384.

\section{Abstract:}

Computer-assisted inspection machines frequently must determine the orientation and location of a plane given set of points that are approximately on the plane. Four commonly used algorithms are analyzed in some detail with particular emphasis on the effects of errors in measuring the data points on the resulting plane. The four algorithms are: The Exact Fit 
algorithm which passes a plane exactly through three data points; the Best of Exact Fits algorithm which selects that plane which gives a best fit to the set of exact fit planes using all combinations of three points selected from the $m(m>3)$ given data points; the Average of Exact Fits algorithm which uses the average of the set of exact fit planes using all combinations of three points selected from the $m(m>3)$ given data points; and the Least RMS plane, that plane which minimizes the RMS of the errors in each of $m(m>3)$ data points. In every case, the effect of errors in the data points takes the form: (error in plane) = (coefficient) $X$ (error in data). The coefficient is a function of the algorithm, the number of data points, and the scatter of these data points. This coefficient is a minimum when the data points are equidistributed on the circumference of a circle. The various algorithms are compared by comparing these coefficients.

\title{
Comments:
}

Low data density was used; most of the fitting approaches are primitive.

\section{Jones,SD; Ulsoy,AG (1995):}

An optimization strategy for maximizing coordinate measuring maching productivity, Part 1: Quantifying the effects of operating speed on measurement quality.

ASME Transactions Journal of Engineering for Industry, 117, 601-609.

\begin{abstract}
:
The ability to quickly obtain precise dimensional information about manufactured parts is vital for any successful mass production operation. Coordinate Measuring Machines (CMMs) can be used to obtain dimensional measurements, but the effects of structural vibrations often limit the speed at which measurements can be obtained. In this, the first of two papers, the effects of operating speed on CMM measurement quality are illustrated, and the factors affecting measurement speed are then analyzed through an Analysis of Variance (ANOVA) study. Results of the illustration and ANOVA show that as operating speed increases, the quality of the measurements obtained decreases, and that the individual axes of the prismatic CMM structure experimentally evaluated react differently to measuring speeds. Also given are results that show the trade-off between measurement time and quality and indicate the existence of optimal operating speeds for certain values of measurement quality. A model is then developed thatdescribes the relationship between measurement speed and measurement quality for use in the optimization strategy presented in Part 2 of this paper.
\end{abstract}




\section{Jones,SD; Ulsoy,AG (1995):}

An optimization strategy for maximizing coordinate measuring maching productivity, Part 2: Problem formulation, solution, and experimental results.

ASME Transactions Journal of Engineering for Industry 117, 610-618.

\section{Abstract:}

In this, the second of two papers focused on optimizing CMM productivity, a strategy is presented for obtaining operating speeds that maximize CMM throughput. The strategy involves posing the objective and constraints in terms of a nonlinear programming problem. The objective is to minimize measuring time, and constraints are on measurement quality, available motor power and other machine specific characteristics. Solutions to various measurement scenarios are given, computed with a generalized conjugate gradient method that uses a golden section acceptable search method. Also, a sensitivity study is presented that utilizes the optimization strategy to determine how change to a CMM's design will affect overall productivity. In the final section, experimental results utilizing the optimally computed trajectories for both single axis and multiple axis motion are given. In addition, an example is presented to demonstrate the implementation of the optimization strategy in an actual measuring application. In the example, a connecting-rod is measured at three speeds: the machine's default speed, a speed constrained by available motor power, and the optimized speed (constrained additionally by measurement quality). The experiment demonstrates a 27 percent reduction in time for the optimized speed compared to the default speed using the Bartlett test for comparing variances in the dimensional data obtained.

\section{Jung,C-Y; Oh,J-H (1991):}

Improvement of surface waviness by cutting force control in milling.

Int. J. Mach. Tools Manufact. 31(1), 9-21.

\section{Abstract:}

To improve the surface waviness in peripheral milling, the feedrate was controlled so that the cutting force measured in the normal direction to the workpiece was constant. A discrete time first order model between the feed rate and the tool deflection was derived with consideration for the machine tool compliance. The model was used for the feedrate control by a fixed gain proportional plus integral (PI) controller. It has been shown by analysis that the tool deflection is directly related to the feedrate and that the tool deflection greatly affects the surface waviness during cutting. The experimental results show that the surface waviness was drastically reduced by the proposed method. 


\section{Kaiser,MJ (1995):}

The geometry of the minimum radial separation functional: the planar case.

J. Meas. Sci. Technol. 6, 1442-1450.

\section{Abstract:}

The minimum radial separation measure associated with a planar simple polygon is computed using an exhaustive search strategy based on the definition of the measure. The algorithm is simple and direct, based on purely geometrical arguments, and provides an alternative and useful way to examine the minimum radial separation measure. The approach of considering the radial separation functional as the basic element of analysis complements rather than replaces existing numerical techniques to find the separation centre point, and because of its fundamental character, is also desirable in terms of standardization. Quantitative measures of tolerance specification that arise from this treatment include an intrinsic 'average' out-ofroundness and values for standard deviation. The concavity of the separation functional at the solution minimum is a useful means to quantify tolerance, and contour plots provide constant out-of-roundness regions. A minimum area difference measure is defined, along with its area difference functional, and the geometry of the separation functionals are illustrated by example.

Comments:

Computationally demanding in the form presented.

\section{Kanada,T (1995):}

Evaluation of spherical form errors - Computation of sphericity by means of minimum zone method and some examinations with using simulated data.

Prec. Eng. 17(4, October), 281-289.

\section{Abstract:}

Because of the practical difficulties of measuring whole spherical surface form errors, no concrete three-dimensional (3-D) verification has yet been developed. This article deals with the calculation of the value of spherical form errors; that is, sphericity. The iterative leastsquares method in which the problem is linearized and the minimum zone method in which the downhill simplex method, one of the nonlinear optimization techniques, is applied are considered. The data to be analyzed are not obtained by actual measurement of a spherical surface, because there is no such measuring system in my laboratory, but simulated by applying surface harmonics (Laplace's spherical function) with a computer. Then the 
application conditions for downhill simplex method are investigated. Furthermore, the roundness values of the spherical surface are compared with the sphericity by means of the minimum-zone method.

Kanada,T; Suzuki,S (1993):

Evaluation of minimum zone flatness by means of nonlinear optimization techniques and its verification.

Prec. Eng. 15(2, April), 93-99.

\section{Abstract:}

This article deals with the application of some nonlinear optimization techniques for minimum zone flatness. The convergence criteria of the techniques, namely the downhill simplex method and the repetitive bracketing method, are considered. The least-squares method is also applied, and subsequently the three methods are compared from the viewpoint of computational accuracy. A surface profile measuring system and a noncontact sensor are used to obtain three-dimensional data. The measured data are expressed by means of perspective mapping. Subsequently, the relationship among the above three methods is clarified according to accuracy and efficiency of the computation. Furthermore, some examples of the relationship between the manufacturing method and the flatness value, and the technique of a skilled hand are described.

\section{Comments:}

Data were taken with a capacitive sensor with relatively low spatial resolution.

\section{Kanada,T; Suzuki,S (1993):}

Application of several computing techniques for minimum zone straightness.

Prec. Eng. 15(4, October), 274-280.

\section{Abstract:}

This research deals with the application of several algorithms to calculate the minimum zone straightness. Generally, in the evaluation of the minimum zone value of form errors such as straightness, flatness, roundness, and cylindricity, nonlinear optimization techniques are usually applied. The problem with the nonlinear technique that depends on computing algorithm is that the computing time may be prolonged. Therefore, some linear search techniques that are relatively easy to program are applied for straightness in this article. Furthermore, the problem can also be linearized by considering the characteristics of the 
measured profile for straightness; thus, the reduction of computing time will be achieved. Then, the problems caused by the above consideration are clarified. Consequently, the convergence criteria and comparison of results by means of several computing methods are investigated. The relationship between flatness and straightness values in some machining conditions and the comparison with the least-squares values are studied.

\section{Kanada,T; Tsukada,T (1986):}

Sampling space in discrete measurements of cylindrical form.

Bull. Japan. Soc. Prec. Eng. 20(3, Sept), 165-170.

\section{Abstract:}

Sampling space in a measurement of cylindrical from by means of discrete data is studied from the view points of form errors, required wave length and measuring accuracy. Firstly it is assumed that any profiles of cylinder surface are basically able to be reproduced by linear-interpolating between two adjacent discrete data. The concept of the new sampling space is such that the difference between the original continuous profile and the simulated curve becomes small enough to satisfy a criterion based on the auto-correlation analysis. In this paper, the criterion is examined and the sampling spaces are presented for some cylinder parts.

\section{Comments:}

Point out that both axial and azimuthal nature of errors must be considered in sampling. Use autocorrelation functions to determine error in form deviation as a function of sampling interval.

\section{Kang,TH (1976):}

The effect of chucking methods on roundness error in the boring process.

ASME Trans. J. Eng. Ind., 233-238.

\section{Kang,TH (1978):}

Sources of geometrical error in machining processes.

SME Tech. Paper MR71-263. 


\section{Kim,N-H; Kim,S-W (1996):}

Geometrical tolerances: improved linear approximation of least squares evaluation of circularity by minimum variance.

International Journal of Machine Tools and Manufacturing 36(March), 355.

\section{Kurfess,TR; Banks,DL (?):}

A statistical analysis of cylindricity.

Technical Memo, Carnegie Mellon University, Pittsburgh, PA, 8 pp.

\section{Abstract:}

This technical brief describes the preliminary results of a joint research effort between the Departments of Mechanical Engineering, Statistics, Engineering and Public Policy, and the Engineering Design Research Center at Carnegie Mellon University. It assumes that all measurements are taken with a CMM having known measurement variance in each coordinate axis (this is the variance in repeated touch-off measurements taken at the same spot). One could generalize the discussion to other measurement devices by expressing their readings in three-dimensional coordinates.

\section{Comments:}

Proposes hexagonal packing as an optimum sampling strategy. Alternatively, suggests concentrating on areas of likely imperfection or critical importance. Does not suggest how to achieve this in practice. Proposed least squares fitting to models of increasing complexity until a normal distribution of residuals is achieved, then testing for conformance and confidence interval estimation.

\section{Kurfess,TR; Banks,DL (1990):}

Statistical verification of part geometry.

draft report, Carnegie Mellon University, Pittsburgh, PA, 15 pp.

\section{Abstract:}

Modern coordinate measurement machines have provided industry with new tools for inspecting complex parts. This paper develops statistical procedures that complement these inspection methods, and it ties the conformance problem into the hypothesis testing formalism of conventional statistics. Also, the paper suggests strategies for efficient sampling of the part 
surface, and an implementation of the usual decision-theoretic formulation of the tradeoff between false acceptance and false rejection of part geometries.

\section{Comments:}

Steps in evaluation are: 1) Take data points. Several ad hoc strategies are suggested for point placement. 2) Fit a series of models, starting with ideal figure and proceeding to model deviations. 3) Fit until some likelihood ratio test is satisfied. 4) Place a confidence region on parameters of final model. If the tolerance region violates tolerance specifications, reject the part.

\section{Kyusojin,A; Yuh'ichi,N; Mori,T; Kobayashi,Y; Toyama,A (1986):}

Relation between the number of measured points and error of the estimated roundness.

Bull. Japan. Soc. Prec. Eng. 20(4, December), 225-230.

\section{Abstract:}

It is important to clarify the difference between the true form of the workpiece and the form estimated from a chain of measured points, in the three-coordinate measuring instrument. In this paper, roundness is first of all dealt with. Using the "min-max" method, the evaluation program is made based on the minimum zone conception. Then the roundness is numerically calculated changing the phase of the starting point of the measurement and the sampling interval or the number of measured points. As a result, some remarkable facts have been clarified as follows. The error of the calculated roundness decreases, as the number of measured points increase. When we select the number of measured points as if the form is made only from the highest order of frequency undulations, we obtain the more certainty because the real form is made from some undulations. Namely, the required number of measured points $N$ is given as $N=2.2 \mathrm{M} / \mathrm{sqrt}(\mathrm{G})$, where $M$ is the highest order of frequency undulation, and $\mathrm{G}$ is the error.

\section{Comments:}

Does probe radius correction on basis of a local fit of a parabolic function. 


\title{
Lai,K; Wang,J (1988):
}

A computational geometry approach to geometric tolerancing.

Proc. 16th N. Am. Mfg. Res. Conf., 376-379.

\begin{abstract}
:
The principles of geometric tolerancing are established in the standards proposed by ANSI and ISO, and in other supporting documents. However, the techniques of geometric tolerancing are not fully developed. In this article, the evaluation of straightness and roundness are investigated using a computational geometry approach and new calculation methods are presented. It is shown that the study of computational geometry will benefit the development of geometric tolerancing both in theory and in practice.
\end{abstract}

\section{Comments:}

Methods for minimum zone computation of form on lines \& circles. Consider the point sample only, do not address sampling. May be useful as starting point for planar datum computation.

\section{Le,V-B; Lee,DT (1991):}

Out-of-roundness problem revisited.

IEEE Trans. Patt. Anal. \& Mach. Intel. 13(3), 217-223.

Abstract:

Abstract The out-of-roundness measurement of a circular profile undertakes different schemes depending on the type or center specified. The most common standard recommended by the American National Standards Institute (ANSI) is the minimum radial separation center. In this paper, we introduce another standard, called the minimum area difference center. Although the two centers are different in characteristics, the approach to finding both centers shares many commonalities. We present an $O(n \log n+k)$ time algorithm to compute the minimum radial separation center, and the minimum area difference center of a simple polygon $\mathrm{G}$, where $\mathrm{n}$ is the number of vertices of $\mathrm{G}$, and $\mathrm{k}$ is the number of intersection points of the medial axis (or the nearest neighbor Voronoi polygons of all skeleton region elements) and the farthest neighbor Voronoi diagram of $\mathrm{G}$. 


\section{Comments:}

Proposes minimum area difference center (of two concentric circles) as an alternative to minimum radial separation zone center. Describes an algorithm to compute both. Claim the minimum radial separation and single-circle minimax solutions are equivalent. Point out that the minimum radial separation (MRS) center of a point set is not identical to the MRS center of the parent simple polygon. They do not address the issue of whether all this is significant in practical circularity computations.

Lee, D (1982) :

Medial axis transformation of a planar shape.

IEEE Trans. Patt. Anal. \& Mach. Intel. 4(4), 363-369.

\section{Abstract:}

The medial axis transformation is a means first proposed by Blum to describe a shape. In this paper we present a $0(n \log n)$ algorithm for computing the medial axis of a planar shape represented by an n-edge simple polygon. The algorithm is an improvement over most previously known results in terms of both efficiency and exactness and has been implemented in Fortran. Some computer-plotted output of the program are also shown in the paper.

\section{Lee,GL; Mou,J (1995):}

A method to enhance the quality and reliability of inspection data for precision manufacturing applications.

Proceedings of the 10th Annual Meeting of the American Society for Precision Engineering, Austin, Texas, 96-99.

\section{Abstract:}

Inspection is commonly used to scrutinize the quality of manufactured products against the established standards and specifications. However, the quality and reliability of many inspection processes are contaminated by various uncertainties. Two prominent sources for measurement uncertainties are: a) the imperfection of a measuring device and b) the dimensional deviation and geometric characteristics of a measured feature. Usually, the effect of both types of uncertainties is confounded with one and other. To assure the quality and reliability of any inspection process, measurement uncertainty needs to be addressed for all data acquisition activities. A method is also needed to identify and decouple the effect of confounded uncertainties. If this can be done, then the collected data can be properly adjusted and a more meaningful analysis results can be drawn. In this paper, the issues of 
uncertainty identification for machine calibration and dimension measurement using artifacts with various geometric features are discussed. Analytical models are derived to describe the confounded effect of both types of uncertainties. Finally, a case study is used to illustrate the confounded effect of the measurement uncertainties.

\section{Comments:}

Describe a specific artifact design and measurement protocol. Refer to application in onmachine inspection.

\section{Liang,R; Woo,T (1995):}

Accuracy and time in surface measurement. Part I: Mathematical foundations.

ASME Trans. J. Eng. Ind., to be published.

\section{Abstract:}

Accuracy and time are known to be conflicting factors in measurement. This paper reevaluates the two-dimensional sampling problem for measuring the surface roughness and establishes that an optimal sampling strategy can be obtained by utilizing the point sequences developed in Number Theory. By modelling a machined surface as a Wiener process, the root-mean-square (RMS) error of measurement is shown to be equivalent to the $L(2)$ discrepancy of the complement of the sampling points. It is further shown that this relationship holds for more general surfaces, thus firmly linking the minimum RMS error of the measurement to the celebrated lower bound on L(2) discrepancy asserted by Roth, a 1958 Fields medalist.

\section{Comments:}

Study of 2D sampling for surface roughness. Shows with proper sampling pattern, for Wiener surface, almost quadratic improvement in accuracy vs. $\mathrm{N}^{*}$ time as compared to uniform sampling pattern. Machined surfaces modeled as random processes. Accuracy of measuring other surface models related to discrepancy of sampling a Wiener surface by a constant factor. Paper compares performance of Zaremba sequence with uniform and random sequences. Roughness descriptors categorized as statistical, extreme value and texture descriptors. Statistical descriptors include average \& rms roughness, skew \& kurtosis. Extreme value descriptors include max peak height, max valley depth, max peak-to-valley. Texture descriptor is correlation length. This paper considers statistical descriptors, esp. average roughness. Model roughness as a zero mean random process on the unit square. Problem is to determine sample locations that minimize error in roughness descriptor. Error is expressed in terms of the autocorrelation function of the surface model and the set of data points. Look at three types of Gaussian surface: Wiener, modified Wiener \& isotropic. 
Modified Wiener surface is obtained by boxcar averaging a Wiener surface. Derive a constant relationship between discrepancy and mean square surface roughness.

\section{Liang,R; Woo,T (1995):}

Accuracy and time in surface measurement. Part II: Optimal sampling techniques.

ASME Trans. J. Eng. Ind., to be published.

\section{Abstract:}

While commonly practiced, the uniform and random distribution of sample points are shown to be far from optimal. This part of the paper presents a sequence for measurement and demonstrates by simulation that, for the same level of accuracy, a nearly quadratic reduction in the number of sample points can be achieved.

\section{Liu,S; Li,J; Zou,Z; Li,Y (1995):}

Evaluation of the position error of two-cylinder workpiece applying maximum material principle.

Proceedings of the 10th Annual Meeting of the American Society for Precision Engineering, Austin, Texas, 92-95.

\section{Abstract:}

A new method for the evaluation of the position error of two cylinders' axes applying the maximum material principle (MMP) is presented in this article. The mathematical model considering the two cylinders as a whole and using the least-squares fitting algorithm is established to evaluate various sizes of workpiece by the personal computer precisely and quantitatively. The data are acquired by means of coordinate measuring machine (CMM). Many advantages can be obtained by using this method instead of the comprehensive gage method. Its application in practical measurement can replace the material gage with a virtual one. The spread of the method will be helpful in making the position error evaluation perfect.

\section{Comments:}

Nonlinear least squares solution for a pair of cylinders constrained to have their axes parallel. 


\title{
Liu,S; Li,Y; Zou,Z; Li,J (1995):
}

A new method of evaluating form error of torus.

Proceedings of the 10th Annual Meeting of the American Society for Precision Engineering, Austin, Texas, 88-91.

\section{Abstract:}

Torus parts are widely used in industrial fields. To measure them and evaluate their form errors, the authors put forward a new method, based on the least squares principle in this paper. Through detailed study by theoretical analysis and extensive computer simulation, it is proven that this method is accurate and quick. The method has a great value of practical usage also, for it can be easily adapted to the widely used coordinate measuring machine (CMM) and other special measuring machines.

\section{Comments:}

Describes a method wherein a linearized approximation is solved by standard least squares methods and iteratively refined.

\section{Lotze,W (1982):}

Generalized fitting algorithms in the coordinate measuring techniques in quality control.

Acta IMEKO 1, 279-286.

\begin{abstract}
:
The paper describes the general conditions for fitting algorithms to calculate the parameters of forms elements from measured points in computer supported coordinate measurement. Standardization of these general conditions (not the algorithm itself) would be useful for definition of new form elements in evaluation software, for comparability of results of different measuring instruments as well as for testing and high accuracy.
\end{abstract}

\section{Comments:}

Recommends least-squares minimization of normal deviations. Also recommends data sets for testing. 
Martino,P (1992):

Analysis of complex geometric tolerances by linear programming.

Computers in Engineering 1, 351-361.

\section{Abstract:}

This paper presents an extension of the linear programming tolerance analysis method introduced by Turner. The new techniques are suited to the analysis of complex geometric tolerances: tolerances involving non-planar datums, tolerance material conditions, and datum material conditions. Key features of the new method are * Ability to model a wide range of tolerance datums. * Datums, as well as tolerances, are represented by linear program constraints. * Rigid body motion degrees of freedom, as well as dimensions (or model variables), are linear program variables. * The linear program is solved by a special three phase simplex method. The new techniques are intended to be used in computer aided tolerance analysis systems.

\section{Matsubara,T; Yamamoto,H; Mizumoto,H (1987):}

Study on accuracy in end mill operations (1st report) - stiffness of end mill and machining accuracy in side cutting.

Bull. Japan. Soc. Prec. Eng. 21(2, June), 95-100.

\section{Abstract:}

This paper deals with a theoretical analysis of machining accuracy in end mill operations. Accuracy is defined by the static stiffness of an end mill and the instantaneous cutting force. The static stiffness of an end mill with two helical cutting edges is theoretically analyzed by a transfer matrix method. The instantaneous cutting force generated during cutting is calculated by the instantaneous depth of cut at any point of a cutting edge. The results obtained are as follows: (1) Up milling is better than down milling from the point of view of accuracy. (2) Variations in feed, width of cut and helix angle of the cutting edge have considerable effects on accuracy. However, depth of cut has almost no effect on it in normal operations. (3) Up milling produces a workpiece cross section with a convex shape, while down milling produces one with a trapezoidal shape. 


\title{
McCool,JI (1986):
}

Comparison of models for the contact of rough surfaces.

Wear 107, 37-60.

\begin{abstract}
:
Numerical comparisons of the Greenwood-Williamson (GW) elastic microcontact model with two more general isotropic and anisotropic models suggest that the GW model gives good order-of-magnitude estimates of the number of contacts, real contact area fraction and nominal pressure that result at a given separation of a rough and a smooth flat plane. In effecting the comparisons, the three parameters of the GW model are related to the three spectral moments $\mathrm{m} 0, \mathrm{~m} 2$ and $\mathrm{m} 4$ of an isotropic or equivalently isotropic surface. It is shown that the real contact area at a given mean plane separation depends only on the bandwidth parameter alpha $=\mathrm{m} 0 \mathrm{~m} 4 / \mathrm{square}(\mathrm{m} 2)$, while the elastically supported load depends on both alpha and $\mathrm{m} 2$. On the basis of the comparisons it is suggested that the GW model be adopted by metrologists to relate measured microgeometry to physically more understandable quantities such as contact density, load, real area and plastic contact density. Accordingly, this paper includes an expository presentation of the GW model complete with tables and a detailed numerical example of their use.
\end{abstract}

\section{McCool,J (1986):}

Relating profile instrument measurements to the functional performance of rough surfaces.

ASME Trans., Journal of Tribology, 1-7.

Abstract:

An easily programmed method is proposed for translating the rms height $(\mathrm{Rq})$ and rms slope ( delta q') determined using a profile measuring instrument, into more readily interpreted measures of functional severity such as the density of plastic contacts or the mean real contact pressure The method involves estimation from the ratio $\mathrm{Rq} /\left(\right.$ delta $\left.\mathrm{q}^{\prime}\right)$, of the exponent $\mathrm{k}$ of an assumed powerfunction relation between the profile spectrum and the spatial frequency. Having estimated $\mathrm{k}$, the mean square curvature is computed analytically and used together with $\mathrm{Rq}$ and delta $\mathrm{q}^{\prime}$ to determine the three input variables needed for the Greenwood-Williamson (GW) microcontact model The GW model is then used to compute, as a function of the separation of two rough surfaces, the contact density, the plastic contact density, the mean load per unit area and the mean load per unit of real contact area The mean square curvature estimated in this manner is compared to the directly measured mean square curvature for 12 distinct surface types. The values compared quite favorably ( within 25 percent) for three of the specimens which included a bearing ball and the ground inner ring 
rolling path of a cylindrical roller bearing The discrepancies exceeded a factor of 3 for three other specimens. The microcontact model output computed using both measured and estimated mean square curvature values showed that some output variables, e.g., plastic contact density, are more discrepant than the estimated and measured curvature values. Other output variables of the microcontact model, in particular, the mean real pressure, attenuate the discrepancies The mean real pressures computed using the calculated and measured curvatures, were within 30 percent for all but three specimens. The maximum discrepancy observed was 55 percent. The results are sufficiently encouraging and the methodology so easy to apply, to commend the practice of routinely supplementing profile measurement data with microcontact model output.

\section{Meijer,J; de Bruin,W (1981):}

Determination of flatness from straightness measurements and characterization of the surface by four parameters.

Prec. Eng. 3(1, January), 17-22.

\section{Abstract:}

Since for medium and larger objects there is no usable flatness reference in the sub-micron range, flatness deviations must be found from straightness measurements. The extensive task of coupling the straight reference lines into a reference flat is done using a computer. This simplifies the measuring procedure and offers much statistical information. The method described can be seen as a generalization of the classical 'Union Jack' method. After evaluation, the measured surfaces are described by four independent characteristic parameters having an important practical meaning.

\section{Mestre,M; Abou-Kandil,H (1994):}

Measuring the errors of form of industrial surfaces: prediction and optimization.

Prec. Eng. 16(4, October), 268-275.

\section{Abstract:}

To acquire maximum information on the geometrical errors of industrially made surfaces at a minimum cost, a method for estimating conditional probabilities of a random signal (Bayesian prediction) is applied to three-dimensional metrology. First, a surface is interpolated between data acquired on a coordinate measuring machine (CMM). Then, for a given probability, limit surfaces are computed that bind a region of space containing the known data and the most probable interpolation of the missing data of the surface. These bounds can be treated as the surface; their points can be considered as if they were actual 
CMM data when fitting a tolerance zone or a datum feature to the data. For Bayesian prediction, the basic hypotheses on the signal are stationarity, ergodicity, and gaussian density. Deviations from these hypotheses and their consequences on the prediction are taken into account and corrections are proposed.

\section{Comments:}

Introduces the idea of estimating form by modeling the real surface with an interpolated function bounded by limit surfaces that have a stated probability of containing the actual surface. The paper touches on the effects of surface filtering by the stylus. It does not address the matter of sample point placement. The statistical character of the surface is determined from a $2 d$ profilometer scan. In general, it seems this should be justified for the surface in question.

\section{Morgan,DE; Piazza,JE; Plyler,RE (1984):}

Inherent errors on $\mathrm{X}, \mathrm{Y}, \mathrm{Z}$ coordinate measuring machines on curved surfaces.

Proc. 1984 Ann. Int. Ind. Eng. Conf., Institute of Industrial Engineers, p.400.

\section{Mou,J; Donmez,MA; Cetinkunt,S (1995):}

An adaptive error correction method using feature-based analysis techniques for machine performance improvement, Part 1: Theory derivation.

ASME Transactions Journal of Engineering for Industry 117, 584-590.

\section{Abstract:}

An adaptive error correction method using a feature-based analysis technique for improving the accuracy of CNC machine tools is presented. The method described in this paper emphasizes the integration of the process-intermittent gauging and analysis techniques with information from pre- process characterization and post-process inspection. The proposed method utilizes the information from pre-process characterization, process-intermittent gauging, and post- process inspection to improve machine performance automatically. Algorithms are derived for analyzing the post-process and process-intermittent inspection data to decouple process-related errors from machine errors and for identifying the residual systematic errors. A feature-based analysis technique is developed to relate the dimensional and form errors of manufactured features to the systematic machine tool errors. Inverse kinematics and statistical methods are used to identify and characterize the contribution of each residual error component on imperfect features. Also a recursive tuning algorithm is developed for fine tuning the geometric-thermal model. 
Mou,J; Donmez,MA; Cetinkunt,S (1995):

An adaptive error correction method using feature-based analysis techniques for machine performance improvement, Part 2: Experimental verification.

ASME Transactions Journal of Engineering for Industry 117, 591-600.

\section{Abstract:}

The development and derivation of an adaptive error correction method using an interactive inspection system and feature-based analysis technique for machine performance improvement were presented in Part 1 of this paper. Here, in Part 2, the experimental verification of the derived feature-based residual error models and analysis technique is described in detail. The experimental procedure and results of both the process-intermittent and the post- process gauging are presented. A polynomial regression method is used to derive the parametric functions that represent the deviation of imperfect features from nominal. The output of these polynomial functions is compared with process-intermittent inspection data to determine the machine errors and then to identify the residual systematic errors. Feature-based residual error models are used to correlate the residual errors to the systematic residual error components. Inverse kinematics technique and multiple regression methods are used to identify and characterize contribution of each error component as a function of the machine's temperature profile and nominal position. The residual error components are then combined with the error components identified through the pre-process characterization process to refine the geometric-thermal error model. After the error model is adaptively fine tuned, it can be used more effectively to compensate the machine tool error for more precise manufacturing.

\section{Murthy,T; Reddy,G; Radhakrishnan,V (1983):}

A method for evaluation of elliptical profiles.

Prec. Eng. 5(2), 79.

Murthy,TSR (1982):

A comparison of different algorithms for cylindricity evaluation.

Int. J. Mach. Tool Des. Res. 22(4), 283-292.

\section{Abstract:}

Precise evaluation of form errors of three dimensional surfaces like cylindricity and sphericity require the development of a mathematical model and an appropriate measuring technique. 
Though the form errors are defined by ISO, their measurement and evaluation techniques are not available. An attempt has therefore been made to devise methods for their measurement and evaluation. Methods of measurement and evaluation procedures of spherical surfaces have been developed and reported elsewhere. In this paper some of the algorithms developed at CMTI and else-here are outlined and a comparison made. The algorithms developed are based on the orthographic projection of the axis of the cylinder and normal least squares fit.

\section{Murthy,TSR (1986):}

A comparison of different algorithms for circularity evaluation.

Prec. Eng. 8(1, January), 19-23.

\section{Abstract:}

The measurement and evaluation of circularity of cylindrical components is very important for the majority of cylindrical workpieces used in precision engineering. Further, since the measurement and evaluation of cylindricity is more complex and time consuming, only circularity is evaluated for most applications. The evaluation of circularity from a circularity graph and/or from digital data requires a suitable algorithm. The most commonly used criterion for this has been the least squares criterion. though it is known that this does not necessarily give the best solution. Hence, an attempt has been made in this paper to propose different algorithms and compare them. The algorithms considered are based on the methods of least squares, intuition, general second-degree equation for a circle, best-fit ellipse and simplex search. A comparison of these methods is presented.

\section{Comments:}

Considers only the point-sample data set of the individual part in finding the circular zone of minimum radial separation. Compares least squares circle and ellipse fits with a simplex search as methods of finding center location. Evaluates performance with equiangular-spaced data with 3-lobe deformation. Does not consider partial arcs nor limacon effect. Simplex method usually gives circularity estimates slightly smaller than other methods, but maximum differences tend not to be large $(<10 \%)$ and the authors seem to imply that their search procedure needs to be repeated from several initial guesses to convince them it has found the global minimum. 
Murthy,TSR (1986):

Evaluation of conditional multiple concentric circular arcs.

Prec. Eng. 8(4, October), 227-231.

Abstract:

Measurement and evaluation of circularity of cylindrical components is well established using roundness measuring instruments, but when circular track of a circular groove has to be evaluated for checking the tolerance on the width of the groove, there are no established methods. Separate evaluation of the circularity of the outer and inner track profiles will not result in one unique centre to determine the track width. Problems of this and more complex nature have been faced while evaluating the tool-changer arm-holder tracks of NC machines. Since no methods are available, an attempt has been made to devise methods for such application. Data from co-ordinate measuring machines have been analysed successfully by using the conditional least squares method.

\section{Comments:}

Minimizes total sum of squares of residuals of both point groups relative to their respective radius estimates. Also solves the problem of a nonconcentric pair of tracks of identical nominal width.

\section{Murthy,TSR; Abdin,SZ (1980):}

Minimum zone evaluation of surfaces.

Int. J. Mach. Tool Des. Res. 20, 123-136.

\section{Abstract:}

The surfaces that are obtained by various machining and finishing processes deviate from the specified geometry. The evaluation of such surfaces for straightness, flatness, circularity, etc. are given by ISO in terms of the minimum zone values. But no specific methods to obtain the minimum zone values are available. Hence an attempt has been made in this paper to present different methods developed at Central Machine Tool Institute (CMTI) for such applications. The present paper deals with the evaluation of spherical, cylindrical and flat surfaces etc. in terms of minimum zone deviation. Monte Carlo technique, normal least squares fit, simplex search and spiral search techniques were applied. Monte Carlo, simples and spiral search techniques were found suitable for minimum zone evaluation. 


\section{Comment:}

Compares various algorithms for minimum zone evaluation of various surfaces. Considers only the current point sample. Does not address sampling issue.

\section{Murthy,TSR; Rao,B; Abdin,S (1979):}

Evaluation of spherical surfaces.

Wear 57, 167-184.

Nasson,MA (?):

Accuracy problems in the measurement of spherical and cylindrical radii.

CSDL-P-2823, Charles Stark Draper Laboratory, Cambridge, MA, 13 pp.

\section{Comments:}

Discusses the problem of tolerancing and measuring partial cylindrical and spherical surfaces. Gives good qualitative and mathematical illustrations of the problem. Suggests profile tolerancing as a solution in preference to size and location tolerancing.

\section{O'Rourke,J (1994):}

Computational Geometry in C

Cambridge Univesity Press, Cambridge, xi+346 pp.

\section{Osanna,PH; Durakbasa,NM; Cakmakci,M; Oberlander,R (1992):}

Cylindricity - a well known problem and new solutions.

Int. J. Mach. Tools Manufact. 32(1/2), 91-97.

\section{Abstract:}

The past twenty years have seen a continued increase in importance of computer aided measurement techniques as a means to control industrial production. At the same time the basic definitions governing design, production and quality control of workpieces have undergone first steps of international harmonization end standardization Focal points of interest included the definitions of nominal, real and substitute geometrical features. The 
cylinder is a characteristic example of feature both on the side of design and production as well as on the side of measurement. The application of CAD, CAE and CAM also puts new demands on inspection and testing in computer integrated production processes. A sophisticated measurement technique, among other things, is considered a most crucial requirement for the production of industrial goods of a controlled and optimized quality. The evaluation of measuring data obtained can be done on CAD and CAE computer using different extensive algorithms. So results of various measuring devices can be compared and an optimization is possible. This is shown by the example of cylindrical workpiece features.>

\section{Pawlus,P; Chetwynd,DG (1995):}

Efficient characterization of surface topography in cylinder bores.

Proceedings of the 10th Annual Meeting of the American Society for Precision Engineering, Austin, TX, 396-399.

\section{Comments:}

Discusses application of cumulative power spectra for determining required sampling interval in surface topography.

\section{Peklenik,J (1963):}

Contribution to the theory of surface characterization.

Ann. CIRP 12, 173-176.

Peklenik,J (1967-68):

New developments in surface characterization and measurements by means of random process analysis.

Proc. Instn. Mech. Engrs. 182, 108-126.

\section{Abstract:}

New developments in engineering and science in systems involving surfaces (friction, wear, contact deformation, reflection, electrical conductivity and resistance, heat transfer, tolerances and fits, etc) require more comprehensive characterization of their micro- and rnacrogeometrical texture. Previous experience exhibits the fact that a statistical description of a surface by means of the first and second moment of the ordinate probability density distribution such as c.l.a. or r.m s. and other parameters is not adequate. This paper discusses the more recent developments of surface characterization, considering a two- and/or threedimensional random process by means of the auto- and cross-correlation functions, power 
spectra, and the slope probability distribution parameters. A representative number of surfaces manufactured by a variety of metal-removal processes have been investigated in order to achieve, first, the differentiation of the surfaces with the same c.l.a. and r.m.s. values, and second, the separation of the periodic and random components in the surfaces. It is shown that the new techniques may disclose the differences in the internal structure of the surfaces. The elementary correlation functions, which may describe analytically the types of real surface profiles, are employed for the design of the surface topography system based on the correlation lengths and correlation wavelengths. On a number of practical examples there is shown the applicability of the proposed surface classification which is independent of the surface generation process. The theoretical approach and the experimental confirmation of the conclusions represent basically a new tool for comprehensive surface characterization. However, it should be considered that such a description requires a data requisition and processing by means of digital or special statistical computers. This circumstance does not affect the basic research work, but it is impractical for workshop application. Therefore new parameters and measuring methods have been developed to make the use of more comprehensive surface characterization methods possible in practice.

\title{
Comments:
}

First good treatment of the idea that surface structure can be characterized as a combination of random and systematic (in this case, periodic) variation and that the characterization can be made independent of the machining process.

\section{Phillips,SD; Borchardt,B; Estler,WT (1995):}

The estimation of measurement uncertainty of small circular features measured by CMMs.

NISTIR 5698, National Institute of Standards and Technology, Gaithersburg, MD, August 1995.

\begin{abstract}
:
This paper examines the measurement uncertainty of small circular features as a function of the sampling strategy, i.e., the number and distribution of measurement points. Specifically, we examine measuring a circular feature using a three-point sampling strategy in which the angular distance between the points varies from widely spaced, 120 degrees, to closely grouped, a few degrees. Both theoretical and experimental results show that the measurement uncertainty is a strong function of the sampling strategy. The uncertainty is shown to vary by four orders of magnitude as a function of the angular distribution of the measurement points. A conceptual framework for theoretically estimating the measuring uncertainty is described and a good agreement with experiment is obtained when the measurements are consistent with the assumptions of the theoretical model.
\end{abstract}


Comments:

Considers a situation in which point sample uncertainty is the only significant error source.

Phillips,SD (1995):

Performance Evaluations.

Chap. 7. In: Coordinate Measuring Machines and Systems. (Ed: Bosch,John) Marcel Dekker Inc., 137-226.

\section{Abstract:}

The subject of coordinate measuring machine (CMM) evaluation is a broad and multifaceted one. The theme of this chapter is the evaluation of CMM measurement uncertainty which is central to the concept of traceability described in chapter 6 . Uncertainty is described using the terminology developed by the International Committee for Weights and Measures (CIPM) [1], which has been recommended by the International Organization for Standardization (ISO) and by national laboratories including the National Institute of Standards and Technology (NIST) [2]. This chapter elucidates the sources of uncertainty and discusses methodologies of how CMM performance can be quantified. This naturally leads to a review of various national and international standards regarding CMM performance evaluation. The chapter ends with a discussion of methods that users can consider when estimating CMM measurement uncertainty using the CIPM formulation. This information will aid CMM users and purchasers when evaluation generalized claims made regarding CMM accuracy. In keeping with the spirit of this book; namely, that it should be an educational resource to the CMM user as rather than an academic review of the literature, only references which are of direct utility to the typical user are cited.

\section{Comments:}

Straightforward \& simple presentations of common error sources in CMM measurement.

\section{Poon,C; Sayles,R (1994):}

Contact analysis of a smooth ball on an anisotropic rough surface.

ASME Trans. J. Tribology 116(October), 850-859.

\section{Abstract:}

The effects of surface roughness and waviness upon the real contact areas, gaps between contact spots, and asperity contact pressures were studied. The distribution of real areas, 
gaps, and contact pressures are presented for different surface roughness, sigma and correlation lengths, beta*. The load-area relationship is compared to Bush's model of a strongly anisotropic rough surface contact using a stochastic approach.

\section{Preparata,FP; Hong,SJ (1977):}

Convex hulls of finite sets of points in two and three dimensions.

Comm. ACM 20(2), 87-93.

\section{Abstract:}

The convex hulls of sets of $\mathrm{n}$ points in two and three dimensions can be determined with $\mathrm{O}(\mathrm{n}$ $\log n$ ) operations. The presented algorithms use the "divide and conquer" technique and recursively apply a merge procedure for two nonintersecting convex hulls. Since any convex hull algorithm requires at least $0(n \log n)$ operations, the time complexity of the proposed algorithms is optimal within a multiplicative constant.

\section{Preparata,FP; Shamos,MI (1985):}

Computational Geometry - An Introduction

Springer-Verlag, New York, xiv+398 pp.

\section{Raja,J (1983):}

Field testing of machine tool diagnostic techniques using surface metrology.

Ann. CIRP 32(1), 503-506.

\section{Abstract:}

Surfaces generated by machine tools are finger print of the machine and tool combination. Any change in the condition of either should be reflected in the component produced specially in the nature of component surfaces generated and its dimensions. If the variables involved in the process like cutting tool, workpiece material and cutting conditions are standardized then the variation in the condition of the machine alone would affect the component produced. The changes in the component surfaces and dimensions could then be used to diagnose the condition of the machine tool. This paper summarizes the results of field trials of this type of diagnostic technique applied to CNC milling machine. 


\section{Comments:}

Uses roundness tester and profilometer data on machined surfaces to determine condition of machine tool

\section{Requicha,AAG (1983):}

Toward a theory of geometric tolerancing.

Int. J. Robotics Res. 2(4), 45-60.

Abstract:

Manual drafting is rapidly being replaced by modern, computerized systems for defining the geometry of mechanical parts and assemblies, and a new generation of powerful systems, called geometric (solid) modeling systems (GMSs), is entering industrial use. Solid models are beginning to play an important role in off-line robot programming, model-driven vision, and other industrial robotic applications. A major deficiency of current GMSs is their lack of facilities for specifying tolerancing information, which is essential for design analysis, process planning, assembly planning for tightly toleranced components, and other applications of solid modeling. This paper proposes a mathematical theory of tolerancing that formalizes and generalizes current practices and is a suitable basis for incorporating tolerances into GMSs. A tolerance specification in the proposed theory is a collection of geometric constraints on an object's surface features, which are two-dimensional subsets of the object's boundary. An object is in tolerance if its surface features lie within tolerance zones, which are regions of space constructed by offsetting (expanding or shrinking) the object's nominal boundaries.

\section{Comments:}

Good review of state-of-art (as of publication date) on shortcomings of solid modeling systems wrt GD\&T. Points out necessity of general \& formal math models for GD\&T parameters. Interpretation of conventional tolerances leads to ambiguities (implied datums, etc.). Size is a difficult issue, esp. in presence of form errors. Propose an offsetting technique to generate bounding surfaces, that are always well-defined. Discusses the problem of partitioning a real part into features; invokes a "slow variation" assumption. 


\title{
Requicha,AAG (1993):
}

Mathematical meaning and computational representation of tolerance specifications.

Proceedings of the 1993 International Forum on Dimensional Tolerancing and Metrology 27, 61-68.

\begin{abstract}
:
The meaning of tolerance specifications must be defined mathematically, to avoid ambiguous interpretations and to provide a sound basis for assessing the correctness of measurement techniques and algorithms. This paper surveys critically the various approaches to tolerancing proposed to date. It classifies these approaches at several levels. Syntactically, it distinguishes between dimensional limits and geometric tolerance specifications; semantically, it considers shape and pose (location and orientation) parameterization versus tolerance zones; and it contrasts worst-case, deterministic approaches with their statistical (stochastic) counterparts. The principles of the offset zone theory of tolerancing, proposed over a decade ago, are presented, to show that there are general approaches, with firm mathematical foundations, which can capture the meaning of geometric tolerances. To support automatic tolerance analysis and other important applications, tolerances must be associated with surfaces and other geometric entities within unambiguous computer representations for mechanical parts (i.e., solid models). Tolerances attached to graphic entities such as lines in a computer-generated drawing do not provide a suitable basis for supporting applications that go much beyond drafting. The paper closes by showing that relatively simple data structures can be used to computationally represent geometric tolerances. These structures can be associated with both of the two most common solid modeling schemes, boundary representation and constructive solid geometry (CSG).
\end{abstract}

\section{Roy,U (1993):}

Computational methodologies for computer aided inspection.

Proceedings of the 2nd Industrial Engineering Research Conference, 466-470.

\section{Abstract:}

The objective of this paper is to discuss the criteria for assessing geometric characteristics of manufactured parts, and the development of systematic procedures and algorithms for comparing measured geometric data from the manufactured parts (features) with specified design tolerances. Though the standards have used the concepts of the tolerance zones (TZs) and minimum zones (MZs) for evaluating different geometric (e.g. form, location, etc.) characteristics, the incompleteness in their definitions create ambiguities in implementing the inspection procedures in coordinate measuring machines (CMM). In this paper the concepts 
of TZ and MZ has been thoroughly investigated for establishing practical methodologies for part inspection.

\section{Roy,U (1995);}

Computational methodologies for evaluating form and positional tolerances in a computer-integrated manufacturing system.

International Journal of Advanced Manufacturing Technology 10(2), 110-117.

Abstract:

In any manufacturing process, the manufactured parts always show some kind of variation in their geometric characteristics (e.g., form, size, orientation, and position). To achieve the desired part quality, these geometric characteristics need to be maintained within predetermined design limits as defined by tolerances. After a part is manufactured it is then inspected to tolerance specifications. In industries, coordinate measuring machines (CMMs) and vision systems are widely used to accomplish the verification tasks. Owing to the lack of proper definitions and implementational details of the correct assessment criteria for different geometric characteristics, it is difficult to develop appropriate algorithms for the inspection software of the CMMs. Though the standards have used the concepts of the tolerance zones (TZs) [1] and minimum zones (MZs) [2] for other geometric (e.g. form, location, etc.) characteristics, the incompleteness in their definitions creates ambiguities in implementing the inspection procedures. It is necessary to develop rigorous definitions of the geometric characteristics (of manufactured parts) that are to be established from discrete, manufactured data points in terms of either TZs or MZs. It is also important to establish the required mathematical criteria for assessing those geometrical characteristics of the manufactured features. In this paper the concepts of $\mathrm{TZ}$ and $\mathrm{MZ}$ have been thoroughly investigated for establishing practical methodologies for part inspection.

\section{Roy,U; Liu,CR; Woo,TC (1991):}

Review of dimensioning and tolerancing: representation and processing.

Comput.-Aided Des. 23(7), 466-483.

\section{Roy,U; Zhang,X (1992):}

Establishment of a pair of concentric circles with minimum radial separation for assessing roundness error.

Comput.-Aided Des. 24(3), 161-168. 
Roy,U; Zhang,X (1992):

Relating CMM data to design specification.

ASQC Annual Quality Congress Transactions 46, 116-123.

\begin{abstract}
:
The use of the coordinate measuring technique in coordinate measuring machines (CMMs) is a common practice in assessing the geometric characteristics of manufactured parts, but due to lack of unique and unambiguous definitions of geometric tolerances and the implementation details of their assessment criteria, it is becoming increasingly difficult to carry out the assessment tasks. The objectives of this paper are to (i) determine unique and unambiguous definitions of the tolerance of any feature, (ii) establish a definition of the measured sizes of all features, (iii) determine the criteria for establishing datums from measured data points of the measured features, and finally (iv) establish computational techniques for assessing the CMM data against the design specification. The computational geometry based techniques have been exploited to establish the ideal geometric features (as per the design specifications) required in the assessment problem as per the standards. The methodology has been implemented and the example has been presented to validate the computational effectiveness of the approach in the case of the roundness error.
\end{abstract}

\title{
Roy,U; Zhang,X (1994):
}

Development and application of Voronoi diagrams in the assessment of roundness error in an industrial environment.

Computers and Industrial Engineering 26(January), 11-26.

\begin{abstract}
:
This paper discusses a robust, computational geometry based technique to establish the roundness error of a measured workpiece in an industrial environment. For evaluating the roundness error, a mathematical formalization has been developed. The properties of a convex hull and the nearest and farthest Voronoi diagrams have been exploited to establish the ideal geometric figures required in the assessment problem as per the standards. The methodology has been implemented and the examples have been presented to validate the computational effectivness of the approach. The proposed method can be easily adopted and installed in the coordinate measuring machines (CMMs) and other computer aided measuring instruments for ready industrial application.
\end{abstract}


Shelton, R (1995):

The realities of data density and its effect on CMM measurement.

SME Workshop on Precision Metrology with CMMs and Applying Imaging and Sensoring Technology to CMM Applications, Indianapolis, IN, June 6-7, 9 pp.

\section{Comments:}

Argues from data, mostly taken on real planar machined surfaces, that high data density is required to accurately recover geometric parameters. Assumes no prior characterization of surface or production process.

\section{Shen,Y-L; Duffie,NA (1995):}

An uncertainty analysis method for coordinate referencing in manufacturing systems.

ASME Trans. J. Eng. Ind. 117(February), 42-48.

\section{Abstract:}

Accurate and consistent transformations between design and manufacturing coordinate systems are essential for high quality part production. Fixturing and coordinate measurement are common coordinate referencing techniques which are used to locate points or measurement points on workpiece reference surfaces to establish these coordinate transformations. However, uncertainty sources such as geometric form deviations in workpiece surfaces, tolerances on fixture locators, and errors in coordinate measurements exist. A result is that coordinate transformations established using the locating and measurement points are inherently uncertain. An uncertainty analysis method for coordinate referencing is presented in this paper. The uncertainty interval concept is used to describe essential characteristics of uncertainty sources in coordinate referencing and coordinate transformation relationships. The method is applied to estimating uncertainties in simple and compound coordinate transformation obtained using coordinate referencing in an experimental mold manufacturing system. Results of Monte Carlo simulations are used to show that the uncertainty analysis method gives a consistent and high percentage of coverage in evaluating coordinate referencing in the examples studied.

\section{Comments:}

Explores the uncertainties in transformations between coordinate systems, e.g. design coordinate system and measuring machine coordinate system or design coordinate system and fixturing coordinate system. Uncertainties include form errors, variations in fixture locating points, measuring system uncertainties. Considers both random and systematic errors. 
Calculations are given for uncertainty in point transformations, inverse and compound transformations.

Shen,Y-L; Duffie,NA (1991):

Uncertainties in the acquisition and utilization of coordinate frames in manufacturing systems.

Ann. CIRP 40(1), 527-530.

\section{Abstract:}

Accurate and consistent transformations between design and manufacturing coordinate frames are essential for precision part production. These coordinate transformations establish surface position and orientation relationships as manufactured parts pass from design through production processes to inspection and surface error analysis. Unfortunately, these coordinate transformations can be uncertain due to factors such as geometric form errors in workpiece reference surfaces, fixture locating point variations, and coordinate measurement errors. A method for representing and estimating these uncertainties and their propagation through multi-stage manufacturing processes is presented in this paper. The method is applicable to systems with combinations of fixturing and coordinate measurements.

\section{Sherrington,I; Smith,EH (?):}

The significance of surface topography in engineering.

Prec. Eng., 79-87.

All machining processes leave characteristic topographic features on the surfaces of components. This paper reviews a wide range of examples in which the surface topography of a component has been found to bear a significant effect on its function. Problems in manufacture which are related to surface roughness requirements are also discussed.

\section{Shunian,Y; Zhu,L; Guangying,L (1988):}

Statistical inferences of form error distribution function.

Prec. Eng. 10(2, April), 97-99.

Abstract:

The concept of statistical evaluation of form errors is illustrated and it is suggested that the form errors can be treated as stochastic variables which fit beta-distributions within arbitrary intervals. Results are in good agreement with experimental values. 


\section{Comments:}

Try to make the case that form error of a feature results from the combined effect of many random variables and that therefore if the effect of each factor is independent, uniform \& small, the distribution of form errors can be treated as normal. Failure to meet these assumptions leads to non-normal distributions which can be fitted with beta-distributions. The authors had good success with beta-distributions on ground and turned cylindrical surfaces with roughness $<1$ and $<10$ micrometer, respectively.

\section{Shunmugam,MS (1986):}

On assessment of geometric errors.

Int. J. Prod. Res. 24(2), 413-425.

\section{Abstract:}

Measurements are carried out on engineering components and analysed to check the conformity of the components to specification. This paper discusses different methods that are useful for assessing the errors on the dimensions, form and position of geometric features. A new approach called the median technique, which gives minimum values of errors is introduced.

\section{Comments:}

Introduces the "median technique", which is said to give minimum values for errors. Stated objective is to arrive at a substitute geometry (based on measurements) which has the least possible deviation from the actual feature. Discusses estimation of size, form and location. Use small numbers of points in their algorithm comparison and do not consider adequacy of the sample. Construct a median figure based on a pair of (nonparallel) ideal bounding figures and evaluate errors relative to that. Some of their search techniques to find the points that define the bounding figures seem suspect. Their examples rely on linearized, rather than normal, deviations. 


\title{
Shunmugam,MS (1987):
}

Comparison of linear and normal deviations of forms of engineering surfaces.

Prec. Eng. 9(2, April), 96-102.

\section{Abstract:}

Form errors of engineering components are usually evaluated using linear deviations. although sometimes. normal deviations are also considered. This paper compares linear and normal deviations, using least squares and minimum deviation techniques.

\section{Shunmugam,MS (1987):}

New approach for evaluating form errors of engineering surfaces.

Comput.-Aided Des. 19(7, July), 368-374.

\begin{abstract}
:
Manufactured features generally deviate in size, form and relations with respect to other features from the features desired by the designer. Designers specify certain limits for these deviations that depend on functional requirements. The specification of different form errors is dealt with by the International Organization for Standardization (ISO). ISO also specifies that an ideal geometric feature must be established from the actual measurements such that the maximum deviation between it and the actual feature concerned is the least possible value. In practice, the least squares (LS) method is sometimes used for establishing the ideal feature, as this method is based on sound mathematical principles. However, the least squares procedure does not yield a minimum value. Therefore some attempts have been made to arrive at a form error based on the minimum deviation $(\mathrm{MD})$ principles. A stray peak or valley on the actual feature introduces considerable variation in the results obtained by the minimum deviation method. This paper suggests a new approach based on the minimum average deviation (MAD). In this method, the ideal feature is established by using a search technique for different geometric features such as straight lines, circles, planes, cylinders and spheres. Using simple numerical examples, the values obtained by this approach are compared with the values obtained by the least squares and minimum deviation methods.
\end{abstract}




\section{Shunmugam,MS (1991):}

Establishing reference figures for form evaluation of engineering surfaces.

Journal of Manufacturing Systems 10(4), 314-321.

Abstract:

A generalized algorithm is presented in this paper to establish the reference figures as specified in the international and national standards on form errors. This algorithm is guaranteed to give optimal results and is well suited for implementation on a computer interfaced, form-measuring instrument or a coordinate measuring machine.

\section{Shunmugam,MS (1991):}

Criteria for computer-aided form evaluation.

ASME Trans. J. Eng. Ind. 113(May), 233-238.

\section{Abstract:}

This paper deals briefly with the concept of form measurement and brings out the different criteria for form evaluation of engineering surfaces. A new criterion based on the minimum average deviation has been suggested. The geometrical forms, namely straight line, circle, plane, and cylinder, are analyzed. Simple numerical examples are included in this paper to illustrate the different methods.

\section{Comment:}

Treats form evaluation as an unconstrained nonlinear optimization problem. Simplex search is used as the minimization technique.

\section{Sourlier,D; Bucher,A (1995):}

Surface-independent, theoretically exact bestfit for arbitrary sculptured, complex, or standard geometries.

Prec. Eng. 17(2, April), 101-113.

Abstract:

In the coordinate measuring technique (CMT), an unambiguous mathematical criterion such as Gaussian least squares sum of departures measured perpendicularly to the calculated 
substitute feature ensures that the results of a geometry bestfit will be independent of the algorithm used. Mathematical methods solving the bestfit problem strictly according to this precisely defined minimizing criterion are available only for a limited number of commonly used standard elements. The proposed algorithm provides this bestfit on any parametrically represented surface $x(u, v, p)$ even if the normally required, special implicit representation $\mathrm{f}(\mathrm{x}, \mathrm{p})=0$ is missing. On the other hand, when applied to any standard element, it provides the classical results. The possibility of using the same, unmodified algorithm for a whole variety of parametrically describable surfaces, comprising complex/sculptured surfaces as well as standard surfaces, allows obvious simplification and standardization of bestfit procedure. The relevance of the proposed algorithm is also made greater by the fact that parametric description is in practice a supposition for automatic measuring/manufacturing as well as for $\mathrm{CAD}$-directed representations, and for that reason $\mathrm{CAD} / \mathrm{CAM} / \mathrm{CAQ}$-data-interchange standards are often based on this mathematical form. In this respect, it serves as a link between CAD-directed automatic.measuring/manufacturing and the evaluation concepts of the classical coordinate measuring technique.

\section{Srinivas,YL; Dutta,D (1995):}

Cyclides in geometric modeling: Computational tools for an algorithmic infrastructure.

J. Mech. Des. 117(September), 363-373.

\section{Srinivasan,N; Farabaugh,F; Wilhelm,RG; Hocken,RJ (1995):}

Correlation models for improved machine tool accuracy.

Proceedings of the 10th Annual Meeting of the American Society for Precision Engineering, Austin, TX, 424-427.

\section{Comments:}

Describes a method for determining machine tool errors by measuring dimensional and form errors of manufactured features.

\section{Stout,KJ; Davis,EJ; Sullivan,PJ (1990):}

Atlas of Machined Surfaces.

Chapman \& Hall, London. 
Stout,KJ; Sullivan,PJ (1989):

The analysis of the three-dimensional topography of the grinding process.

Ann. CIRP 38(1), 545-548.

\section{Summerhays,KD; Henke,RP; Baldwin,JM; Cassou,RM; Finch,JK; Detry,R (1994):}

Dimensional inspection techniques specification - Planar face and datum features with addendum on opposite parallel plane features.

R-95-QAP-01.,University of San Francisco, 2130 Fulton St., San Francisco, CA 94117-1080

\section{Abstract:}

The use of coordinate measuring machines (CMMs) and other discrete point sampling devices for the dimensional inspection of part surfaces has raised questions regarding the proper interpretation of data which technically constrains the surface only at the points measured. Reconciling the intrinsic limitations of such inspection techniques with dimensioning and tolerancing standards which often make the implicit assumption that surfaces can be fully characterized has been an issue of major concern in the metrology community in recent years. In addition economic considerations argue for more efficient and reliable procedures for dimensional inspection. This has led to the desire to find sampling and data analysis methods by which the information available through discrete point sampling is maximized. The Dimensional Inspection Techniques Specification (DITS) project addresses the uncertainty inherent in the current methods (or lack of methods) for choosing the number and distribution of data points and appropriate fitting criteria required for efficient and effective inspection of manufactured parts. This report summarizes work carried out in Phase II of DITS, treating single face and datum features and includes work commissioned as an addendum to the priginal project. The additional study has as its focus opposite parallel plane features. The general thrust of the effort here has been to recognized an important distinction in the treatment of deviations from nominally planar form. A certain portion of such deviation is unquestionably best treated as being of a random or pseudo-random nature. But the most commonly encountered analysis algorithms in current software systems of CMMs and related devices are based on the notion that all of the deviation is of this type. In so far as discrete sampling devices cannot provide more than an estimate of the full surface character, it is important to have as much additional information as possible about anticipated systematic form deviations of the part surfaces to be inspected. Such information can serve to inform the sampling and analysis processes, leading to a more effective quality assurance program. Early in the course of the work reported here, an extensive literature survey was conducted to gather as much information as was available about the typical nonidealities associated with various manufacturing methods for the production of planar surfaces. In addition a wide array of parts was gathered from various sources. These parts, of many different materials 
and representing the most common production techniques, were all accurately and densely sampled on high quality CMMs. This data served first as a basis for study of the two traditional data analysis methods for planar surfaces: the simple linear least squares method (which we designated LSO) and the minimum zone method (which we have abbreviated MZ0). Through treatment of the dense sample sets and a myriad of subsets of increasing sparse character, we mapped the limits of these approaches. The dense sample sets served another important purpose: providing a basis for the development of a modeling system for smoothly varying systematics. We founs that most parts could be quite reasonably approximated by polynomial surfaces of relatively low order. It was also noted, especially for milled surfaces, that more abrupt form deviations were not uncommon. Often these were of the step type. Consideration of smoothly varying systematic deviations from planarity led to the development of two new algorithms for data analysis. For each a companion sampling strategy was devised. We have called the new methods Extended Least Squares (LSF) and Extended Zsone (EZN). The associated sampling optimization methods are designated as LSAMP and ESAMP, respectively. The LSAMP method uses a priori knowledge of systematic form deviations to determine a pattern for sampling the surface in a way which, for a given number of sample points, provides as accurate an assessment of the average planar substitute geometry (in the least squares sense) as possible and a good estimate of the true standard deviation about that plane. In the LSF analysis then, the standard deviation of this sparse sample set serves to establish confidence limits on the estimated part surface. The ESAMP method uses a priori kmowledge of systematic form deviations to determine a pattern for sampling the surface in a way which, for a given number of sample points, provides as accurate a basis as possible for the fitting of a specified surface form of higher order than a simple plane. In the EZN analysis then, this higher order fitted surface and its standard deviation serve to establish confidence limits on the estimated part surface. EZN then yields a substitute planar geometry by applying the minimum zone method to this estimated surface. Through the inspection of lot-size quantities of real parts, and by using extensive surface simulations, we have established the superiority of these methods over the traditional approaches. We find that ESAMP/EZN is especially useful for surfaces having low to moderate proportions of random form deviation. LSAMP/LSF may be preferred when the proportion of random character is relatively high. The methods described have been extended to the treatment of slots. There a maximum (rather than minimum) zone is used in the EZN method, providing a basis for the estimation of size, orientation and location. Again lot-size quantities of real parts were produced and densely sampled in order to provide a basis for the testing of these algorithms. And again noteworthy improvements in accuracy over traditional analysis methods have been achieved. In modeling surfaces which show systematic form deviations such as steps, or other discontinuous behavior not readily modeled by polynomial surfaces, we have employed methods of factor analysis for the determination of eigen surfaces. These then serve as a means of representing to LSAMP and/or ESAMP the anticipated nature of common form deviations. Obviously the use of these algorithms requires some advance knowledge of part variability. The better one understands his prroduction, the more effective these methods will be in application. But even the simple assumption that one's parts may manifest some systematic second order character (i.e. 
concavity, convexity, or twists) can be enough to gain advantage over methods which ignore such considerations. The approach taken here has yielded results of significant utility in the measurement of single planar surfaces and of opposite parallel plane features. There is good reason to expect that similar treatment of other feature forms will provide advantages over traditional methods as well.

\section{Summerhays,KD; Henke,RP; Baldwin,JM; Cassou,RM (1996):}

Dimensional Inspection Techniques Specification. Phase IVA. Full Inside-Diameter Cylindrical Features(Aluminum). Artifact Analysis of Cylinders Produced by Center Drill/Drill Method.

\section{Summerhays,KD; Henke,RP; Baldwin,JM; Cassou,RM (1996):}

Dimensional Inspection Techniques Specification. Phase IVA. Full Inside-Diameter Cylindrical Features(Aluminum). Artifact Analysis of Cylinders Produced by Center Drill/Drill/Ream Method.

\section{Summerhays,KD; Henke,RP; Baldwin,JM; Cassou,RM (1996):}

Dimensional Inspection Techniques Specification. Phase IVA. Full Inside-Diameter Cylindrical Features(Aluminum). Artifact Analysis of Cylinders Produced by Center Drill/Drill/Bore/Ream Method.

\section{Summerhays,KD; Henke,RP; Baldwin,JM; Cassou,RM (1996):}

Dimensional Inspection Techniques Specification. Phase IVA. Full Inside-Diameter Cylindrical Features(Aluminum). Artifact Analysis of Cylinders Produced by Center Drill/Drill/Plunge End Mill Method.

\section{Summerhays,KD; Henke,RP; Baldwin,JM; Cassou,RM (1996):}

Dimensional Inspection Techniques Specification. Phase IVA. Full Inside-Diameter Cylindrical Features(Aluminum). Artifact Analysis of Cylinders Produced by Center Drill/Drill/Contour End Mill Method.

\section{Summerhays,KD; Henke,RP; Baldwin,JM; Cassou,RM (1996):}

Dimensional Inspection Techniques Specification. Phase IVA. Full Inside-Diameter Cylindrical Features(Aluminum). Literature Survey and Bibliographic Data Base. 


\section{Summerhays,KD; Henke,RP; Cassou,RM; Baldwin,JM; Brown,CW (1995):}

New algorithms for the evaluation of discrete point measurement data and for sample point selection on surfaces with systematic form deviations.

Proceedings of the 10th Annual Meeting of the American Society for Precision Engineering, Austin, TX, 388-391.

\section{Comments:}

Description of DITS phase II \& III results.

\section{Sweet,AL; Noller,D; Lee,S-H (1985):}

Statistical design for the location of planes and circles when using a probe.

Prec. Eng. 7(4, October), 187-194.

\section{Abstract:}

A statistical approach to the problem of establishing the location of planes and circles by use of a touch-trigger probe mounted on a machining centre is presented. Equations for determining the minimum number of measurements to make and their location in order to locate a part within a specified confidence interval are given. An explicit solution to the parameter estimation problem for the circle is derived, and its properties are compared with solutions from two other methods. It is shown that one of the methods has an estimator whose accuracy does not improve as the number of measurements made is increased because the probe can move only along Cartesian axes when it is mounted on a machining centre.

\section{Comments:}

Their model assumes random and uncorrelated measurement and form errors with zero mean. Machine positioning errors are assumed negligible. 


\section{Taylor,WB (?):}

A software approach to establishing a primary datum plane.

internal report, Westinghouse Electric Corp., Sunnyvale, CA, 14 pp.

Abstract:

A mathematical description of the criteria for establishing a primary datum plane is formulated which conforms to ANSI Y14.5-1982. Computer programs are written, which using this description, establishes the primary datum plane for any number of points in three dimensional space. The plane established using this description is significantly different from those established by commercially available coordinate measuring machine (CMM) software which uses a least squares fitting criteria.

Comments:

Solves the minimax fit to the data set, then uses the outer bounding plane as the datum. Assumes that the available data set represents the surface adequately.

Taylor,WB (?):

Approaches to determining flatness.

internal report, Westinghouse Electric Corp., Sunnyvale, CA, 8 pp.

Comments:

Compares two algorithms for finding the planes of minimum separation about a data set.

Thomas, TR (1981):

Characterization of surface roughness.

Prec. Eng. 3(2), 97-104.

Thomas, TR (1982):

Rough Surfaces.

Longman, London. 
Touissant,G (1983):

Computing largest empty circles with location constraints.

Int. J. Comput. Inform. Sci. 12(5), 347-358.

Traband,MT; Joshi,S; Wysk,RA; Cavalier,TM (1989):

Evaluation of straightness and flatness tolerances using the minimum zone.

Manuf. Rev. 2(3, September), 189-195.

Abstract:

This paper presents an algorithm that can be used to determine if sample measurements from a coordinate measuring machine, or other two-or-three-dimensional measuring device, meet the ANSI Y14.5M requirements for straightness or flatness. The procedure utilizes the concept of a convex hull to give the minimum tolerance zone from a set of measured points. To date, approximation methods or iterative search techniques have been primarily utilized for determining the minimum zone, but these methods do not necessarily provide the absolute minimum zone. This paper presents a method that guarantees the minimum zone. Examples are presented which compare the minimum zone method developed with the least-squares evaluation method.

\section{Comments:}

Algorithms for minimum zone flatness and straightness from point data. Uses convex hull on the data set, claims to be superior to approximation of iterative search techniques in that it guarantees correct solution. Includes comparison of minimum zone with least squares results. Acknowledges the sampling issue but does not address it. Straightness: Minimum zone of a point set is the minimum zone of its convex hull. One of the sides of the minimum zone coincides with an edge of the convex hull. Further, only antipodal point pairs will admit parallel lines satisfying the minimum zone requirement. So: 1) Determine convex hull. 2) Find antipodal pairs. 3) For each pair, determine the minimum diameter. 4) Find the minimum of these diameters $=$ the minimum zone. Overall complexity is $O(n \log n)$. Flatness: Analogous procedure on $3 \mathrm{~d}$ convex hull, but without antipodal pair simplification. Complexity $\mathrm{O}\left(\mathrm{n}^{* * 2}\right)$. Discuss possibility of dynamic minimum zone evaluation as data are gathered. Mention the fact that some least squares routines minimize an objective function which involves distances normal to some axis or plane, rather than to the fitted feature. 
Tsukada,T; Anno,Y; Yanagi,K; Suzuki,M (1977):

An evaluation of form errors of cylindrical machine parts by spiral tracing method.

Proc. 18th Int. MTDR Conf., 529-535.

Tsukada,T; Kanada,T (1982):

Measurement of cylindrical form errors using a non-contact detector.

Prec, Eng. 4(3, July), 153-158.

Abstract:

Measurement using a spherical tip stylus cannot give a correct assessment for cylindrical form errors of finely turned parts due to their cutting tool marks. The electrostaticcapacitance type gap sensor applied in this research is useful in eliminating the influence of cutting tool marks, which should be regarded as the surface roughness. The sensor does not damage the cylinder surface, as a stylus might. Further, two measuring methods, a round tracing method and a spiral one, were compared

Authors' concern is that conventional filters (mechanical \& electrical) do not remove surface roughness data in the axial direction. They use a capacitance probe, which takes a 2D average, instead of a physical probe in combination with filtering, which takes a $1 D$ average. They compare a spiral tracing pattern to a series of equally-spaced circular traces. Correct for eccentricity and tilt of specimen relative to rotational axis by least squares fitting. Discuss the effects of sampling pattern and aliasing of tooling pattern on apparent magnitude of Fourier components of error. Article shows planar mappings of cylindricity data as 3D perspective plots and contour plots.

\section{Tsukada,T; Kanada,T; Okuda,K (1984):}

An evaluation of roundness from minimum zone center by means of an optimization technique.

Bull. Japan. Soc. Prec. Eng. 18(4), 317-322. 


\section{Tsukada,T; Sasajima,K (1981):}

A three-dimensional measuring technique for surface asperities.

\section{Abstract:}

Optimization techniques to evaluate the minimum zone cylindricity deviation are presented in this research. Such the direct search methods as the newly improved simplex method and Powell's method are applied and their algorithms are proposed. Consequently, it is made clear that the newly developed algorithm in the improved simplex method is the most effective from the view point of the computation time and the accuracy of cylindricity deviation.

\section{Comments:}

Least squares overestimates form error, minimax is computationally intensive. Authors compare two search methods for determining minimum zone solution. Analyze roundness tester data on 206 surface produced by turning, contour milling \& grinding. Study Powell's method, which is a direct search and two variations of simplex search. All 3 are reported to be capable of reasonable results, one of the simplex methods is fastest. No details of measured surfaces are given.

\section{Tsukada,T; Shukui,L; Kanada,T (1988):}

A verification technique of minimum zone conical taper form.

Bull. Japan. Soc. Prec. Eng. 22(3, September), 211-215.

\section{Abstract:}

This paper proposes a new verification technique for minimum zone value of conical taper form errors. Since the mathematical analysis to achieve the minimum zone evaluation is nonlinear and complex, the simplex method, one of nonlinear optimization techniques, is applied. The convergence criterion is settled to get a solution for this algorithm of iterative computation. Furthermore, the minimum zone values are compared with the least squares values. Consequently, it is made clear that this algorithm is useful, the least squares value frequently over estimates the minimum zone value. Further the difference between the rates of taper in minimum zone and least squares is presented. 


\title{
Vadar,HO; Ozsoy,TM (1992):
}

A method for selecting measurement points on planar surfaces of objects with holes.

Computers in Engineering 1, 429-436.

\begin{abstract}
:
In this paper, a method facilitating the automatic selection of measurement points on planar faces of objects with holes will be presented. The objective is to further automate the process of generation of inspection programs for coordinate measuring machines. The method developed aims for the creation of candidate points on a planar face of an object and the optimal selection of a required number of measurement points among the candidate points. A triangulation technique based on a finite element mesh generation method has been implemented in creating the candidate points. Except for the special case of four points, a random selection process is utilized for choosing among the candidate points. The effects of randomness are compensated by applying a convex hull computation algorithm to find the set of points among other candidate sets that covers the largest area. The method has been successfully incorporated into a software demonstrating the feasibility of the approach.
\end{abstract}

\section{Comments:}

Process is executed in two steps: Set of candidate points is created and require number are selected from set. A triangular mesh is generated on the surface and candidate points are places at the centroids of the triangles. Measurement points are selected either at random or by an optimum distribution search. Suggested strategies for optimum distribution are: 1) Choose points with largest sum of distances from already-chosen points. 2) Choose outermost points (those on convex hull). 3) Repetitive application of 2 after elimination of previously selected points.

\section{Vajapeyam,S; Keefe,M (1992):}

Triangulated surface construction from scattered 3-d points.

Computers in Engineering 2, 29-33.

\section{Abstract:}

A three-dimensional analog to the Gabriel Graph structure is defined and an algorithmic procedure for the construction of a triangulated surface from scattered data points in three dimensions is developed based on the concept on three-dimensional Gabriel Graphs. The algorithm does not require the points to be in the form of a grid or on contours. The closest point 3-D Delaunay triangulation of the points is first constructed and the Delaunay triangles 
that satisfy the Gabriel Graph criterion are identified. From this set of triangles, extraneous triangles are removed, resulting in a triangulated open surface passing through all the given data points. This surface can then be subjected to smoothing algorithms if necessary and a smooth surface of the desired continuity can be constructed using available interpolation techniques. The algorithm can be used for constructing surfaces from scattered data in mechanical design, geographic terrain modeling and modeling biological surfaces from CT scans and MRI scans.

\title{
Comments:
}

Also provides a fairly good survey of previous work on surface construction from 3D data.

\section{Voelker,HB (1993):}

A current perspective on tolerancing and metrology.

Proceedings of the 1993 International Forum on Dimensional Tolerancing and Metrology 27, 49-60.

\begin{abstract}
:
Tolerancing and metrology are primary tools for specifying, assessing, and controlling geometric variability in design and manufacturing. Both evolved from shop and drafting practice and are partially codified in standards. In recent years, the introduction of new technology - notably Coordinate Measuring Machines (CMMs) and CAD systems - exposed gaps, ambiguities, and inconsistencies in current practice. These findings triggered a wave of effort to formalize tolerancing and metrology, by 'mathematizing' the American tolerancing standard and by producing a new companion standard for measurement. These two developments are unlikely to be the end of the story, however, because other gaps and issues remain. Thus tolerancing and metrology currently stand at a watershed: the era of evolutionary practice seems to be ending, and a new era based on mathematically defined techniques and standards seems to be dawning. This paper sets a context for the Proceedings papers that address these matters, by surveying the major approaches to tolerancing and metrology and the issues they engender. The paper concludes with brief discussions of issues not addressed well or at all in the Proceedings, and some speculations on the future.
\end{abstract}

\section{Comments:}

Discusses needed work beyond that currently in progress by Y14.5.1 and B89.3.2. Compares parametric tolerancing methods (worst-case limit, statistical, vectorial) with GD\&T. Gives arguments why parametric tolerancing may remain useful. References a couple of new proposals for tolerancing. Discusses the dogma of process-independent specification relative 
to concurrent engineering. Concise expression of the sampling issue. Simple presentation of coupled constraints in functional tolerancing.

\section{Walker,I; Wallis,AF (1992):}

Applications of 3-d solid modeling to coordinate measuring inspection

Int. J. Mach. Tools Manufact. 32(1/2), 195-201.

\section{Abstract:}

This paper describes a set of algorithms for the automation of component inspection using coordinate measuring machines. Solid modeling techniques are used to describe the component, and this model is then used to generate a set of inspection points and a collisionfree probe path. The model is also used in the analysis of the resulting measurements. The algorithms have been implemented in a system that will handle the range of form, location and attitude tolerances, as defined by BS 308 , for planar, cylindrical, conical and spherical features.

\section{Comments:}

Considers requirements on a system to completely automate inspection by CMM. 1) Shape description, including GD\&T. They use CSG with tolerances attached as attributes. 2) Sample selection. Generate a pattern of points on surfaces of a faceted representation \& select from that set. Point density is user specified, point selection/face is random. Reachability is determined. 3) Path generation. 4) Analysis \& reporting.

\section{Walker,RK; Srinivasan, V (1993):}

Creation and evolution of the ASME Y14.5.1M standard.

Proceedings of the 1993 International Forum on Dimensional Tolerancing and Metrology 27, 19-30.

\section{Abstract:}

The forthcoming ASME Y14.5M-1993 standard will soon have a mathematical companion in the form of ASME Y14.5.1M-1993 standard. Effort on this mathematical document began in early 1989. This paper describes the origin, workings, and accomplishments of the Y14.5.1 subcommittee that was responsible for this new standard. 


\section{Wang,Y (1992):}

Application of optimization techniques to minimum zone evaluation of form tolerances.

Winter Annual Meeting of the American Society of Mechanical Engineers, Anaheim, CA, USA, Nov 8-13.

\section{Abstract:}

This paper presents a method for determining form tolerances of a machined part with sampled measurement points from a coordinate measuring machine. The method follows the definitions of the geometric tolerances used in the current ANSI standards. Evaluation of the minimum tolerance zone is formulated as a constrained optimization problem. It is demonstrated that a general-purpose algorithm for constrained nonlinear optimization can be effective in determining minimum tolerance zones. Test cases are given to illustrate successful applications of the method to form features of straightness, flatness, roundness, and cylindricity. This method is compared with the least square evaluation method. Furthermore, special structures of the problem are discussed to show that they can be exploited to facilitate more efficient and reliable algorithms.

\section{Weckenmann,A; Eitzert,H; Garmer,M; Weber,H (1995):}

Functionally-oriented evaluation and sampling strategy in coordinate metrology.

Prec. Eng. 17(4, October), 244-252.

\section{$\underline{\text { Abstract: }}$}

This is a review on the evaluation criteria and sensing strategy used in coordinate metrology today. The necessity of basing future evaluation entirely on the test objective (product function, process control), the evaluation methods (algorithms) this requires, and the needed sampling strategy (including datapoint distribution) are described.

\section{Comments:}

Two goals of production metrology: geometric verification \& process correction. Question applicability of least squares fitting where form errors in part surpass random errors in apparatus. They point out that extant standards require boundary-estimators of substitute geometry. Miss the point that least squares fits can be reasonable estimators of these boundary representations. 


\section{Weckenmann,A; Heinrichowski,M (1985):}

Problems with software for running coordinate measuring machines - The use of virtual volumetric standards.

Prec. Eng. 7(2, April), 87-91.

\section{Abstract:}

The validation of software for coordinate measuring machines is increasingly being seen to be as important as the assessment of the machine construction and its coordinate system. Virtual volumetric standards are the coordinates of points on the surface of an imaginary workpiece which can be used to evaluate $\mathrm{cmm}$ software. This paper gives some examples of virtual volumetric standards and their use.

\section{Comments:}

Primarily discusses the "classical" nonidealities of lines, planes, circles and cylinders.

\section{Weckenmann,A; Heinrichowski,M; Mordhorst,H-J (1991):}

Design of gauges and multipoint measuring systems using coordinate-measuring-machine data and computer simulation.

Prec. Eng. 13(3, July), 203-207.

\section{Abstract:}

The existing coordinate-measuring-machine programs using least square methods for the calculation of geometric elements are not suitable for the evaluation of component fitting. However, evaluation programs that supply statements that are comparable with those of gauge inspection can be used to support the design of gauges and multipoint measuring systems. How to optimize an inspection device by simulation in practice is shown by the example of a forged bevel gear.

\section{Comments:}

There are two methods of gauge simulation. 1) Model the gauge and search for a transformation on the measured points into the gauge coordinate system s.t. no measurement point collides with the gauge. 2) Define the contacting elements of the part and compare their formal parameters with those of the gauge. Include a discussion of sample sufficiency for 
cylindrical features and propose that knowledge of the production process can be used to select an efficient probing pattern; illustrate this with the obvious example of a 3-lobed part.

\section{Weckenmann,A; Mordhorst,H-J (1989):}

Effect of sensing strategy on measurement results in coordinate metrology.

Proceedings of the IMEKO Congress of the International Measurement Confederation, 2, 202-206.

\section{Abstract:}

Coordinate measuring machines are used in production metrology as the standard means of , verifying workpieces. In order to determine geometric characteristics of features, i.e. dimensions, location parameters, orientation parameters and form deviations, points on the surface of a workpiece are sensed and the values of the coordinates of the measuring points determined to allow calculation of the parameters required (e.g. diameter, coordinates of center, distances) as measurement results. The accuracy with which the measured substitute parameters based on the random sample of the selected sensed points describe the complete real shape of the workpiece is examined.

\section{Whitehouse,DJ (1971):}

The properties of random surfaces of significance in their contact.

Ph.D. Dissertation, U. of Leicester.

\section{Whitehouse,DJ (1982):}

The parameter rash - Is there a cure?

Wear 83, 75-78.

\section{Abstract:}

In recent years there has been a proliferation of parameters with which to specify surface texture. Some of these parameters are useful, but most are not. The result of this rash is confusion and expense. In this paper the cause of the growth of such parameters is identified and ways of minimizing it are explored.

\section{Comments:}

Standardization without research has contributed to the problem. 
Woo,T (1993):

Hammersley sampling for efficient surface coordinate measurements.

Proc. NSF Des. \& Mfg. Sys. Conf., 2, Charlotte, NC.

Woo,TC; Liang,R (1993):

Dimensional measurement of surfaces and their sampling.

Comput.-Aided Des. 25(4, April), 233-239.

\section{Abstract:}

The number of the discrete samples for the dimensional measurement of machined surfaces and their coordinates is investigated. Counter to intuition, there need not be quadratically more samples than in the case for sampling lines or curves. To justify this novel scheme, accuracy is defined as the discrepancy of a finite point set. Then, from number theory, a particular sequence of numbers is used to compute the sampling coordinates resulting in a number that is linear in $1 \mathrm{D}$, at the same level of accuracy that is achieved by a $2 \mathrm{D}$ uniform distribution. Finally, experimental results of the measurement of machined surfaces modeled as random processes are compiled.

\section{Comments:}

Consider number and placement of points required to characterize a machined surface. Crystal-clear discussion of fitting and choice of metric. Method of selecting sampling points presumes no knowledge of systematic deviations from ideal form. Presumes random form deviations in the machined surfaces. To normally-considered sources of methods divergence (sampling, fitting) they add another (distance). Introduce the concept of accuracy as expressed by the discrepancy of a finite set of points. Compare sampling based on the Hammersley sequence with uniform sampling and find nearly quadratic reduction in number of samples required for a given accuracy. Fitting is considered as an elevation in dimensionality; points are $0 \mathrm{D}$; fitting curves or surfaces raises the dimensionality of the construct, reducing data storage requirements. Also, group behavior emerges, aiding interpretation. Consider three basic criteria for fitting data to ideal geometries: extreme (inside, outside), through, offset (e.g. minimum zone). Therefore, a (linear or nonlinear) distance function is minimized wrt an ideal surrogate geometry. Surrogate geometry and ideal (nominal) geometry are then compared using some distance function. Discrete sampling is an approximation process. For an infinite sample, the error of approximation is zero. For a given sample size, how small can the error be? Where should the samples be located? Consider a unit square bounded in each dimension by $(0,1)$. Discrepancy of a set of $\mathrm{N}$ points is a quantity with a lower bound that depends only on $\mathrm{N}$ and $\mathrm{d}$ (dimensionality). Simulate 
machined surfaces by two random process models: Wiener \& Gaussian. No results on actual machined surfaces.

\section{Worring,M; Smeulders,AWM (1995):}

Digitized circular arcs: characterization and parameter estimation.

IEEE Trans. Patt. Anal. \& Mach. Intel. 17(6, June), 587-598.

\section{Abstract:}

The digitization of a circular arc causes an inherent loss of geometrical information. Arcs with slightly different local curvature or position may lead to exactly the same digital pattern. In this paper we give a characterization of all centers and radii of circular arcs yielding the same digitization pattern. The radius of the arcs varies over the set. However, only one curvature or radius estimate may.be assigned to the digital pattern. We derive an optimal estimator and give expressions for the bound on the precision of the estimation. This bound due to digitization is the deterministic equivalent of the Cramer/Rao bound known from parameter estimation theory. Consider the estimation of the local curvature and local radius of a smooth object. Typically such parameters are estimated by moving a window along the digital boundary. Methods in the literature show a poor precision in estimating curvature values, relative errors of over $40 \%$ are often found. From the definition of curvature it follows that locally the curve can be considered a circular arc and hence the method presented in this paper can be applied to the pattern in the window giving estimates with optimal precision and a measure for the remaining error. On the practical side we present examples of the residual error due to the discrete grid. The estimation of the radius or curvature of a circular arc at random position with an estimation window containing 10 points (coded with nine Freemancodes) has a relative deviation exceeding $2 \%$. For a full disk the deviation is below $1 \%$ when the radius $r$ exceeds four grid points. The presented method is particularly useful for problems where some prior knowledge on the distribution of radii is known and where there is noise-free sampling.

\section{Xiong,YL (1990):}

Computer aided measurement of profile error of complex surfaces and curves: theory and algorithm.

Int. J. Mach. Tools Manufact. 30(3), 339-357.

\section{Abstract:}

This paper is concerned with two key problems for computer aided measurement of profile error of surfaces and curves: (1) evaluation algorithm of profile error on the basis of 
minimum zone principle or maximum material condition; (2) computer aided arbitrament for minimum zone principle and maximum material condition. A general mathematical model for computer aided measurement of profile error of any surface and curve is proposed and the united algorithm for evaluation and arbitration criterion are derived on the basis of this model. Finally, we deal with error separation and the influence of probe radius as well as control strategies for quality assurance.

\section{Comments:}

Includes examples for ellipsoid, sphere, paraboloid, ellipse, circle, parabola, line, other figures.

\section{You,SJ; Eman,KF (1989):}

Scallop removal in die milling by tertiary cutter motion.

ASME Trans. J. Eng. Ind. 111(August), 213-219.

\section{Abstract:}

The feasibility of a newly proposed method for the removal of cutter marks in die finishing operations has been investigated. The method is based on the superposition of a tertiary motion onto the conventional cutter motions. Path equations that consider motion and geometric variables have been derived and used as a basis for the development of a computer simulation model for generating three-dimensional surface representations. It has been shown that the tertiary motion results in a considerable reduction in the volume of the material in the scallops or blend marks left on the surface.

\section{Zhang,GM; Kapoor,SG (1991):}

Dynamic generation of machined surfaces, part 2: Construction of surface topography.

ASME Trans. J. Eng. Ind. 113(May), 145-153. 


\section{Zhang,X; Roy,U (1993):}

Criteria for establishing datums in manufactured parts.

Journal of Manufacturing Systems 12(1), 36-50.

\section{Abstract:}

This paper identifies some of the problems and ambiguities with existing standards for establishing datums in manufactured components. Most of the criteria as described in the standards do not uniquely define the datums from measured data points of manufactured features. Three important characteristics need to be validated in establishing datums. By using the computational geometry-based techniques and least squares method, it is possible to provide unique and unambiguous criteria for establishing datums. 


\section{UNLIMITED RELEASE}

\section{INITLAL DISTRIBUTION}

Kim D. Summerhays

Harney Science Center, Room 409

University of San Francisco

2130 Fulton Street

San Francisco, California 94117-1080

MS9001 T. O. Hunter, 8000

Attn: MS9005 J. B. Wright, 2200

MS9006 E. E. Ives, 5200

MS9004 M. E. John, 8100

MS9054 W. J. McLean, 8300

MS9007 R. C. Wayne, 8400

MS9002 P. N. Smith, 8500

MS9405 T. M. Dyer, 8700

MS9141 L. A. Hiles, 8800

MS9133 J. M. Baldwin, 8220 (20)

MS9003 D. L. Crawford, 8900

MS9133 K. A. Hontz, 8220

MS9133 R. D. Pilkey, 8220

MS9420 L. A. West, 8200

Attn: MS9405 R. E. Stoltz, 8202

MS9409 L. N. Tallerico, 8204

MS9133 B. E. Affeldt, 8206

MS9104 M. H. Rogers, 8210

MS9403 J. M. Hruby, 8230

MS9409 A. J. West, 8240

MS9133 J. A. Fordham, 8240-1

MS9409 R. H. Stulen, 8250

MS9405 Manager, 8220

V. C. Barr, 8250-1

MS 9021 Technical Communications Department, 8535, for OSTI (10)

MS 9021 Technical Communications Department, 8535/Technical Library, MS 0899, 13414

MS 0899 Technical Library, 13414 (4)

MS 9018 Central Technical Files, 8523-2 (3) 


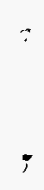

